\title{
Contribution to the Knowledge of the Phytocenotic Diversity of the Lesser Antilles Revisiting Some Old and More Recent Floristic Data
}

\author{
Philippe Joseph, Jean-Philippe Claude, Kévine Baillard, Yelji Abati, \\ Yanis Jean-Francois, Péguy Major, Jean-Emile Simphor, Jean-Valéry Marc, \\ Séverine Ely-Marius, Stéphane Sophie
}

UMR ESPACE DEV, University of the French Antilles, Schœlcher, Martinique

Email: joseph.phil@wanadoo.fr

How to cite this paper: Joseph, P., Claude, J.-P., Baillard, K., Abati, Y., Jean-Francois, Y., Major, P., Simphor, J.-E., Marc, J.-V., Ely-Marius, S. and Sophie, S. (2020) Contribution to the Knowledge of the Phytocenotic Diversity of the Lesser Antilles Revisiting Some Old and More Recent Floristic Data. Open Access Library Journal, 7: e6191.

https://doi.org/10.4236/oalib.1106191

Received: February 26, 2020

Accepted: March 24, 2020

Published: March 27, 2020

Copyright $\odot 2020$ by author(s) and Open Access Library Inc.

This work is licensed under the Creative Commons Attribution International License (CC BY 4.0).

http://creativecommons.org/licenses/by/4.0/

\section{(c) (i) Open Access}

\begin{abstract}
The complex structure of the abiotic factors in the Lesser Antilles leads to singular vegetation ranging from the dry to the humid. Geomorphology by means of the wide variety of topographic facets is the determining parameter which leads to gradients of mesological factors. Among the latter, both on the windward and leeward facades, the precipitation is distinguished by bioclimatic staging associated with plant stagings which consist of a mosaic of phytocenoses of variable sizes, ages, floristic compositions and structures as well as of different architecture. Based on examples of old and recent floristic surveys by authors which indicate ecotones, types and inversions of vegetation, we have shown the great phytocenotic diversity of the plant cover in the Lesser Antilles which are all biosystemic responses to the effects of natural and anthropogenic hazards. Thanks to this significant ecosystemic plasticity, the Lesser Antilles represent veritable laboratories for autoecological and synecological studies as well as of floristic succession.
\end{abstract}

\section{Subject Areas}

Ecology, Ecosystem Science, Plant Science, Biodiversity, Biogeography

\section{Keywords}

Lesser Antilles, Bioclimates, Phytocenoses, Ecosystemic Potentialities, Types of Vegetation, Plant Dynamics, Anthropisation 


\section{Introduction}

On a global scale, vegetations are mosaics of various specific units, ages, structures and compositions which belong to different stages of the plant succession and which show the structural variability of the mesological factors [1]-[12]. Anthropisation seems to be a factor in increasing biocenotic biodiversity [13] [14] [15] [16] [17]. The insular Caribbean is also affected by this global phenomenon of the humanisation of biotopes. Indeed, since the takeovers of the 17th century up to the present day, the groundcovers of the Lesser Antilles have been subject to the various effects in terms of intensity and frequency, of human activities [18] [19] [20]. The great diversity of the floristic formations constitutes one of the unique characteristics of the current flora of the Antilles [21] [22] [23] [24]. As a consequence, these islands represent a veritable laboratory for the synchronic study of the vegetation and therefore the landscapes [25] [26] [27]

[28]; Figure 1). In what follows, we will show, using a few examples, the diversity of the physiognomic types which reflect a great specific and morphogenetic diversity. This summary is the result of studies based on qualitative indicators and constitutes a preliminary work ahead of more quantitative approaches.

\section{Materials}

With respect to their size and their geomorphology, the Lesser Antilles exhibit great variability [29] [30] [31] [32]. There are the low-lying islands, the low islands and the highland islands. These different groups consist of plural topographic facies which contribute to the great diversity of the biotopes. The peaks, the ridges, the valleys or dales, the flats, the plateaus, the plains and the rocky ledges are all topographic facets which modify, to a variable degree, the structure

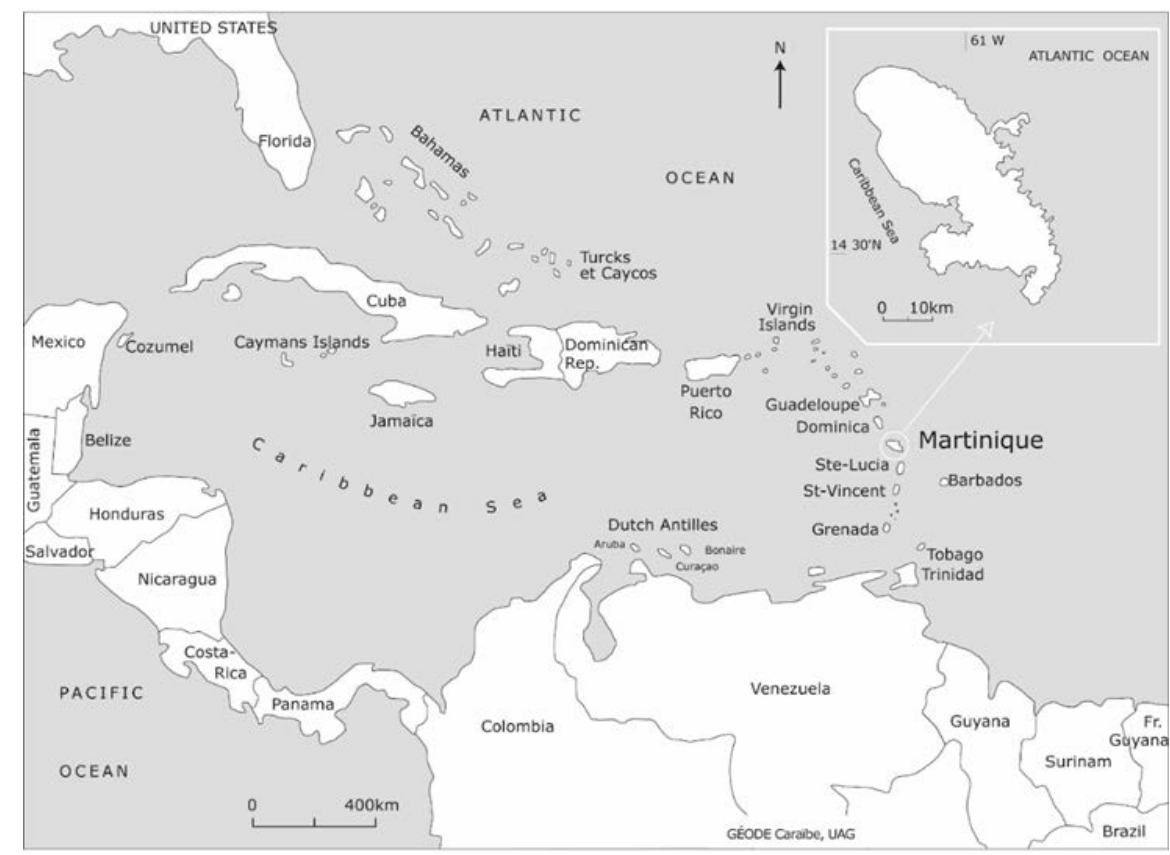

Figure 1. The Lesser Antilles in the Caribbean. 
of the factors of the macroclimate [33] [34] [35]. Consequently, the latter are signified by differentiated altitudinal gradients. The result of all this is many microclimates and mesoclimates which lead to great specific and phytocenotic diversity. Precipitation is the key mesological factor, particularly concerning the mountainous Lesser Antilles. On these islands, the orographic rains delimit a bioclimatic staging which defines a plant staging which is therefore phytocenotic. From the coast to the summit, we can identify hyper-humid, humid, humid subhumid and dry subhumid bioclimates which typically give rise to the following forest vegetation: mountain ombrophilous, sub-mountain ombrophilous, seasonal evergreen types and seasonal evergreen in their xeric facies. This above-mentioned general outline makes it possible to identify the floristic potentialities of the low-lying islands.

\section{Methods}

To highlight the diversity of the phytocenoses and associated floristic corteges, we have taken certain data from our predecessors into account as well as our own [36] [37] [38] [39] [40]. These data come from inventories within minimum areas ranging from $400 \mathrm{~m}^{2}$ (plant stages subject to the dry subhumid bioclimate) to more than $2000 \mathrm{~m}^{2}$ (plant stages influenced by the humid bioclimate). When the eco-climatic conditions are homogeneous, these minimum areas correspond to the smallest surface areas providing the maximum of information on the structure and evolution of the phytocenoses and therefore on the ecological profiles of the taxa. Due to the differences in the surface area of inventories and the methods of the floristic surveys, the data generated by the above-mentioned authors were considered to be non-compliant. Consequently, for the purposes of the analysis and on the basis of these biodemographic data, we have constructed indicators of qualitative abundance [41]: (++++++: 500 individuals): very high abundance/(+++++: 100 individuals): high abundance/(++++: 50 individuals): medium abundance/(+++: 25$)$ : low abundance/very low abundance (++: 15): negligible $(+: 6)$. The results are presented in two parts. In the first part, the ratios of abundance and by inference of dominance between taxa are indicated using tables summarising the data from different authors. However, the old data are presented in light of the conceptual framework of modern ecology. In the second part, in order to compare the stations with respect to the population structure of the species, we performed a CFA (Correspondence Factor Analysis) and an AHC (Ascending Hierarchical Classification) using the XLSTAT software based on a contingency table composed of the stational average floristic abundances. In the tables the strata correspond to different classes of heights.

\section{Results}

\subsection{Examples of Pre-Sylvatic, Young Structured and Secondary Sylva Groupings Inventoried from 1938 to the Present Day}

\subsubsection{Saint-Kitt-Nevis (J. S. Beard, 1949)}

The author does not specify the ratios of significance between the different spe- 
cies; however, the physiognomic description of this grouping seems to correspond to an organisation where the woody tree is ecologically predominant. Table 1 refers to a tropical seasonal evergreen plant formation of lower horizon, degraded and located at the pre-sylvatic or young sylvatic stage.

\subsubsection{Montserrat (J. S. Beard, 1949)}

In Table 2, the most abundant trees are Lonchocarpus violaceus, Bursera simaruba, Tabebuia heterophylla. Under this discontinuous structure, in unequal proportions, Bourreria succulenta, Guaettarda scabra, Acacia sp, Croton flavens, Haemotoxylum campechianum, Citharexylum spinosum form a matrix of non-stratified associations. The structural and architectural elements of Table 2 indicate that this grouping is in the presylvatic or young sylvatic evolutionary stage.

As regards Table 3, Canella winterana, Coccoloba pubescens, Zanthoxylum monophyllum, Zanthoxylum punctatum are structuring agents of an open tree phytocenosis with which dominate low xerophilous species, variously associated such as Opuntia dillenii, Melocactus intortus, Croton sp, Agave sp, Pilosocereus roynei, Comocladia dodanea, Clerodendrum aculeatum. In Table 3, this tropical seasonal evergreen floristic grouping of lower horizon in its most xeric facies corresponds to the preforest stage.

Table 1. Tropical seasonal evergreen unit of lower horizon.

\begin{tabular}{|c|c|c|c|c|c|}
\hline Plant species & Abundance & $\begin{array}{c}\text { Stratigraphic } \\
\text { levels }\end{array}$ & Plant species & Abundance & $\begin{array}{c}\text { Stratigraphic } \\
\text { levels }\end{array}$ \\
\hline Acacia ssp & + & $S 3$ & $\begin{array}{c}\text { Lonchocarpus } \\
\text { pentaphyllus }\left(^{*}\right)\end{array}$ & +++ & $\mathrm{S} 2 / \mathrm{S} 1$ \\
\hline $\begin{array}{l}\text { Bourreria } \\
\text { succulenta }\end{array}$ & + & $S 3$ & $\begin{array}{c}\text { Pisonia } \\
\text { suborbiculata }\end{array}$ & + & $S 3$ \\
\hline $\begin{array}{c}\text { Capparis } \\
\text { cynophallophoa }\end{array}$ & + & $S 3$ & $\begin{array}{c}\text { Pithecellobium } \\
\text { unguis-cati }\end{array}$ & + & $S 3$ \\
\hline $\begin{array}{c}\text { Chrysobalanus } \\
\text { icaco }\end{array}$ & + & $S 3$ & Plumeria alba & + & $S 3$ \\
\hline $\begin{array}{l}\text { Coccoloba } \\
\text { diversifolia }\end{array}$ & +++ & $S 2 / S 1$ & Prosopis juliflora & + & S3 \\
\hline $\begin{array}{l}\text { Coccoloba } \\
\text { pubescens }\end{array}$ & +++ & $S 2 / S 1$ & Randia aculeata & + & S3 \\
\hline Croton flavens & + & $S 3$ & $\begin{array}{l}\text { Sideroxylon } \\
\text { salicifolia }\end{array}$ & ++ & $\mathrm{S} 2 / \mathrm{S} 3$ \\
\hline Ficus trigonata & + & $S 3$ & $\begin{array}{c}\text { Tabebuia } \\
\text { heterophylla }\end{array}$ & +++ & $\mathrm{S} 2 / \mathrm{S} 1$ \\
\hline Guettarda scabra & ++ & $\mathrm{S} 3 / \mathrm{S} 2$ & & & \\
\hline
\end{tabular}

$(++++++)$ : very high Abundance/ $(++++)$ : high Abundance/ $(+++)$ : Medium abundance/(++): low Abundance/(+): very low Abundance. $\left({ }^{*}\right)$ : given the bioclimatic stage, it is highly plausible that it is Lonchocarpus violaceus, however stational conditions may allow for the installation of the species cited by the author. S1: Upper strata/S2: Middle strata/S3: Lower strata. 
Table 2. Tropical seasonal evergreen formation of lower horizon.

\begin{tabular}{|c|c|c|c|c|c|}
\hline Plant species & Abundance & $\begin{array}{c}\text { Stratigraphic } \\
\text { levels }\end{array}$ & Plant species & Abundance & $\begin{array}{c}\text { Stratigraphic } \\
\text { levels }\end{array}$ \\
\hline Acacia sp. & ++ & $\mathrm{S} 2 / \mathrm{S} 3$ & Inga laurina & + & S2 \\
\hline $\begin{array}{c}\text { Annona } \\
\text { squamosa }\end{array}$ & + & S3 & Jacquinia armilaris & + & S3 \\
\hline $\begin{array}{l}\text { Bourreria } \\
\text { succulenta }\end{array}$ & +++ & $\mathrm{S} 2 / \mathrm{S} 3$ & Leucaena leucocephla & +++ & $\mathrm{S} 1 / \mathrm{S} 2$ \\
\hline Bursera simaruba & ++++ & $\mathrm{S} 2 / \mathrm{S} 1$ & Lonchocarpus violaceus & ++++ & $\mathrm{S} 2 / \mathrm{S} 1$ \\
\hline $\begin{array}{l}\text { Capparis } \\
\text { flexuosa }\end{array}$ & + & S1 & Malpighia linearis & + & S3 \\
\hline $\begin{array}{l}\text { Citharexylum } \\
\text { spinosum }\end{array}$ & +++ & $\mathrm{S} 1 / \mathrm{S} 2$ & Myrcia citrifolia & ++ & S3 \\
\hline $\begin{array}{c}\text { Coccoloba } \\
\text { diversifolia }\end{array}$ & ++ & $\mathrm{S} 1 / \mathrm{S} 2$ & Pilosocereus royeni & + & S3 \\
\hline Croton astroides & + & S1 & Pisonia subcordata & +++ & S3 \\
\hline Croton flavens & +++ & S1 & Plumeria alba & + & $\mathrm{S} 3 / \mathrm{S} 2$ \\
\hline $\begin{array}{l}\text { Erytroxylon } \\
\text { havanense }\end{array}$ & ++ & S1 & Randia aculeata & +++ & S3 \\
\hline $\begin{array}{l}\text { Exostema } \\
\text { caribaeum }\end{array}$ & ++ & $\mathrm{S} 1 / \mathrm{S} 2$ & Tabebuia heterophylla & ++++ & $\mathrm{S} 1 / \mathrm{S} 2$ \\
\hline Guettarda scabra & ++ & $\mathrm{S} 1 / \mathrm{S} 2$ & Tamarindus indica & + & S2 \\
\hline $\begin{array}{l}\text { Haemotoxylum } \\
\text { campechianum }\end{array}$ & ++ & $\mathrm{S} 1 / \mathrm{S} 2$ & Tecoma stans & ++ & S3 \\
\hline
\end{tabular}

(++++++): very high Abundance/(++++): high Abundance/(+++): medium Abundance/(++): low Abundance/(+): very low Abundance. S1: Upper strata/S2: Middle strata/S3: Lower strata.

Table 3. Highly xeric littoral facies where xeromorphosis is pronounced.

\begin{tabular}{|c|c|c|c|c|c|}
\hline Plant species & Abundance & $\begin{array}{c}\text { Stratigraphic } \\
\text { levels }\end{array}$ & Plant species & Abundance & $\begin{array}{c}\text { Stratigraphic } \\
\text { levels }\end{array}$ \\
\hline Agave $s p$ & + & $S 3$ & $\begin{array}{l}\text { Melocactus } \\
\text { intortus }\end{array}$ & + & S3 \\
\hline Canella winterana & +++ & $S 2 / S 3$ & Opuntia dillenii & + & S3 \\
\hline $\begin{array}{l}\text { Clerodendrum } \\
\text { aculeatum }\end{array}$ & ++ & $S 3$ & Pilosocereus roynei & + & S3 \\
\hline $\begin{array}{l}\text { Coccoloba } \\
\text { pubescens }\end{array}$ & ++++ & $S 2 / S 1$ & Prosopis juliflora & + & S3 \\
\hline $\begin{array}{c}\text { Comocladia } \\
\text { dodanea }\end{array}$ & ++ & $S 3$ & $\begin{array}{l}\text { Zanthoxylum } \\
\text { monophyllum }\end{array}$ & ++++ & $\mathrm{S} 2 / \mathrm{S} 3$ \\
\hline Croton $s p$ & ++ & $S 3$ & $\begin{array}{l}\text { Zanthoxylum } \\
\text { punctatum }\end{array}$ & ++++ & $\mathrm{S} 2 / \mathrm{S} 3$ \\
\hline
\end{tabular}

(++++++): very high Abundance/(++++): high Abundance/(+++): medium Abundance/(++): low Abundance/(+): very low Abundance. S1: Upper strata/S2: Middle strata / S3: Lower strata.

\subsubsection{Dominique (J. S. Beard, 1949)}

The species in Table 4 form a low-stratified preforest eco-unit. However, Tabebuia heterophylla, Byrsonima spicata, Lonchocarpus violaceus, Coccoloba 
Table 4. Highly degraded pre-forest facies.

\begin{tabular}{|c|c|c|c|c|c|}
\hline Plant species & Abundance & $\begin{array}{c}\text { Stratigraphic } \\
\text { level }\end{array}$ & Plant species & Abundance & $\begin{array}{c}\text { Stratigraphic } \\
\text { level }\end{array}$ \\
\hline Ardisia obovata & + & S3 & $\begin{array}{l}\text { Haematoxylon } \\
\text { campechianum }\end{array}$ & +++ & $\mathrm{S} 3 / \mathrm{S} 2$ \\
\hline Byrsonima lucida & + & S2 & $\begin{array}{c}\text { Lonchocarpus } \\
\text { violaceus }\end{array}$ & +++ & $\mathrm{S} 2 / \mathrm{S} 1$ \\
\hline Byrsonima spicata & ++ & $\mathrm{S} 2 / \mathrm{S} 1$ & Myrcia citrifolia & ++ & S3 \\
\hline Calliandra tergemina & + & S3 & Pimenta racemosa & + & $\mathrm{S} 2 / \mathrm{S} 3$ \\
\hline Capparis flexuosa & + & S3 & Pisonia fragrans & ++ & $\mathrm{S} 2 / \mathrm{S} 3$ \\
\hline $\begin{array}{c}\text { Chamaecrista } \\
\text { glandulosa var. } \\
\text { swartzii }\end{array}$ & + & S3 & Randia aculeata & + & S3 \\
\hline Coccoloba pubescens & +++ & S2/S3 & Rhyticocos amara & + & $\mathrm{S} 2 / \mathrm{S} 3$ \\
\hline Croton ssp & ++ & S3 & $\begin{array}{c}\text { Tabebuia } \\
\text { heterophylla }\end{array}$ & +++ & S2/S3 \\
\hline Erithalis fruticosa & + & S3 & $\begin{array}{c}\text { Tetrazygia } \\
\text { angustifolia }\end{array}$ & + & S3 \\
\hline Garcinia humilis & + & $\mathrm{S} 2 / \mathrm{S} 3$ & $\begin{array}{l}\text { Zanthoxylum } \\
\text { punctatum }\end{array}$ & ++ & S3 \\
\hline Guettarda scabra & ++ & $\mathrm{S} 3 / \mathrm{S} 2$ & & & \\
\hline
\end{tabular}

$(++++++)$ : very high Abundance/(++++): high Abundance/(+++): medium Abundance/(++): low Abundance/(+): very low Abundance. S1: Upper strata/S2: Middle strata/S3: Lower strata.

pubescens and Pisonia fragrans, although peaking at low heights, dominate the other taxa. The populations of the latter are characterised by a high density of individuals. The resulting structure is very heterogeneous from a synecological point of view: it equates to a dense mosaic of plant associations, most of whose representatives belong to the physiognomic class of nanophanerophytes.

\subsubsection{Sainte-Lucie (J. S. Beard, 1949)}

This plant cortege from Table 5 is in the preforest stage and is typical of the tropical seasonal evergreen flora of lower horizon and xeric facies within this example the most abundant trees being Amyris elemifera, Guettrada scabra and Citharexylum spinosum.

In Table 6 only the dominant taxa are presented and form a grouping at the secondary forest stage.

\subsubsection{Saint-Vincent (J. S. Beard, J. P. Fiard, P. Joseph)}

\section{1) J. S. Beard (1949)}

The species in Table 7 make up diverse pre-forest associations. Nevertheless, the observable physiognomies are identical. The predominant plant matrix is made up of bushes that have reached their optimal morphogenetic development and is dotted with trees in the expansion phase such as Amyris elemifera, Citharexylum spinosum, Guettarda scabra, Piscidia carthagenensis. 
Table 5. Highly degraded pre-forest facies.

\begin{tabular}{|c|c|c|c|c|c|}
\hline Plant species & Abundance & $\begin{array}{c}\text { Stratigraphic } \\
\text { level }\end{array}$ & $\begin{array}{c}\text { Plant } \\
\text { species }\end{array}$ & Abundance & $\begin{array}{c}\text { Stratigraphic } \\
\text { level }\end{array}$ \\
\hline Acacia tamarindifolia & ++ & S3 & Croton $s p$ & + & S3 \\
\hline $\begin{array}{c}\text { Aegiphila } \\
\text { martinicensis }\end{array}$ & + & S3 & $\begin{array}{c}\text { Erythroxylon } \\
\text { havanense }\end{array}$ & ++ & S3 \\
\hline Amyris elemifera & +++ & S3 & Guettrada scabra & +++ & $\mathrm{S} 2 / \mathrm{S} 3$ \\
\hline Bourreria succulenta & +++ & S2/S3 & Plumeria alba & + & S2/S3 \\
\hline Calliandra slaneae & + & S3 & Randia aculeata & + & S3 \\
\hline Calliandra tergemina & ++ & S3 & $\begin{array}{l}\text { Ternstroemia } \\
\text { pedoncularis }\end{array}$ & + & S3 \\
\hline $\begin{array}{l}\text { Citharexylum } \\
\text { spinosum }\end{array}$ & +++ & $\mathrm{S} 2 / \mathrm{S} 3$ & & & \\
\hline
\end{tabular}

$(++++++)$ : very high Abundance/(++++): high Abundance/(+++): medium Abundance/(++): low Abundance/(+): very low Abundance. S1: Upper strata/S2: Middle strata/S3: Lower strata.

Table 6. Seasonal evergreen forest units (municipality of Praslin).

\begin{tabular}{ccc}
\hline Plant species & Abundance & Stratigraphic level \\
\hline Eugenia pseudopsidium & ++++ & $\mathrm{S} 2 / \mathrm{S} 1$ \\
Coccoloba pubescens & ++++ & $\mathrm{S} 2 / \mathrm{S} 1$ \\
Maytenus laevigata & +++ & $\mathrm{S} 2 / \mathrm{S} 1$ \\
Tabebuia heterophylla. & +++ & $\mathrm{S} 2 / \mathrm{S} 1$ \\
\hline
\end{tabular}

(++++++): very high Abundance/(++++): high Abundance/(+++): medium Abundance/(++): low Abundance/(+): very low Abundance. S1: Upper strata/S2: Middle strata/S3: Lower strata.

\section{2) Joseph \& J. P. Fiard (1996, King's Hill)}

In view of the qualitative abundance indicators in Table 8, the dominant cortege of this late secondary or subclimactic sylva is, in order of significance, made up of Inga laurina, Pouteria multiflora and Faramea occidentalis. The species of the upper stratum are mainly Hymenaea courbaril, Inga laurina and Pouteria multiflora. This association is somewhat atypical since on the one hand Inga Laurina and on the other hand Hymenaea courbaril and Pouteria multiflora belong distinctly to the secondary and climactic forest stages. As for the middle and lower strata, they are made up of Bursera simaruba, Pisonia fragrans, Chrysophyllum argenteum, Ocotea patens and Faramea occidentalis.

\subsubsection{The Grenadines (R. A. Howard, J. S. Beard \& J. P. Fiard)}

Very small islands, the Grenadines are exclusively under the influence of the dry bioclimate. The main potentiality is tropical seasonal evergreen vegetation of lower horizon and xeric facies. Since this small archipelago is characterised by a variable xericity, it is highly plausible that there once existed the typical semi-deciduous sylvatic climactic type in the tropical dry season. Today's biocenoses present a phenology close to that which characterises deciduous vegetation in the tropical dry season. Defoliation is total and is correlated with a long drought: sometimes more than five months. 
Table 7. Seasonal evergreen forest grouping.

\begin{tabular}{|c|c|c|c|c|c|}
\hline Plant species & Abundance & $\begin{array}{c}\text { Stratigraphic } \\
\text { level }\end{array}$ & Plant species & Abundance & $\begin{array}{c}\text { Stratigraphic } \\
\text { level }\end{array}$ \\
\hline Acacia nilotica & ++ & $\mathrm{S} 3 / \mathrm{S} 2$ & Guettarda odorata & ++ & $\mathrm{S} 3 / \mathrm{S} 2$ \\
\hline Acacia tamarindifolia & + & S3 & Guettarda scabra & ++ & $\mathrm{S} 3 / \mathrm{S} 2$ \\
\hline $\begin{array}{c}\text { Aegiphila } \\
\text { martinicensis }\end{array}$ & + & S3 & Lantana camara & + & S3 \\
\hline Amyris elemifera & ++ & S3 & Lantana involucrata & + & S3 \\
\hline Bourreria succlenta & +++ & S2/S3 & $\begin{array}{l}\text { Morisonia } \\
\text { americana }\end{array}$ & + & $\mathrm{S} 3 / \mathrm{S} 2$ \\
\hline Capparis baducca & + & S3 & $\begin{array}{c}\text { Piscidia } \\
\text { carthagenensis }\end{array}$ & ++ & S2/S3 \\
\hline $\begin{array}{c}\text { Capparis } \\
\text { cynophallophora }\end{array}$ & + & S3 & $\begin{array}{c}\text { Pithecellobium } \\
\text { unguis-cati }\end{array}$ & ++ & $\mathrm{S} 3 / \mathrm{S} 2$ \\
\hline Capparis hastata & ++ & S3 & Plumeria alba & + & S3 \\
\hline Capparis odoratissima & + & S3S & Randia aculeata & + & S3 \\
\hline $\begin{array}{l}\text { Citharexylum } \\
\text { spinosum }\end{array}$ & ++ & $\mathrm{S} 2 / \mathrm{S} 3$ & Rauvolfia viridis & + & S3 \\
\hline Cordia globosa & ++ & $\mathrm{S} 3 / \mathrm{S} 2$ & $\begin{array}{l}\text { Schaefferia } \\
\text { frutescens }\end{array}$ & + & S3 \\
\hline Cordia obliqua & ++ & $\mathrm{S} 2 / \mathrm{S} 3$ & Schoepfia schreberi & + & S3 \\
\hline Erithalis fruticosa & + & S3 & Schoepfia schreberi & + & S3 \\
\hline $\begin{array}{c}\text { Erythroxylon hva- } \\
\text { nense }\end{array}$ & ++ & S3 & Tecoma stans & + & S3S \\
\hline Eugenia monticola & +++ & $\mathrm{S} 3 / \mathrm{S} 2$ & Trichila hirta & + & $3 / \mathrm{S} 2$ \\
\hline
\end{tabular}

$(++++++)$ : very high Abundance/(++++): high Abundance/(+++): medium Abundance/(++): low Abundance/(+): very low Abundance. S1: Upper strata/S2: Middle strata/S3: Lower strata.

Table 8. Seasonal evergreen forest grouping.

\begin{tabular}{ccc}
\hline Plant species & Abundance & Stratigraphic level \\
\hline Bursera simaruba & +++ & $\mathrm{S} 2 / \mathrm{S} 1$ \\
Chrysophyllum argenteum & ++ & $\mathrm{S} 3 / \mathrm{S} 2$ \\
Faramea occidentalis & ++++++ & $\mathrm{S} 3 / \mathrm{S} 2$ \\
Hymenaea courbaril & ++ & $\mathrm{S} 2 / \mathrm{S} 1$ \\
Inga laurina & +++++ & $\mathrm{S} 1 / \mathrm{S} 2$ \\
Ocotea patens & ++ & $\mathrm{S} 3 / \mathrm{S} 2$ \\
Pisonia fragrans & ++ & $\mathrm{S} 3 / \mathrm{S} 2$ \\
Pouteria multiflora & ++++ & $\mathrm{SS} 2 / \mathrm{S} 1$ \\
\hline
\end{tabular}

$(++++++)$ : very high Abundance/(++++): high Abundance/(+++): medium Abundance/(++): low Abundance/(+): very low Abundance. S1: Upper strata/S2: Middle strata/S3: Lower strata.

\section{1) R.A. Howard (1950): Isle of Ronde (examples of woody associations)}

The tree associations of Table 9 are dominated either by Coccoloba venosa, Diospyros inconstans and Citharexylum spinosum and are in the pre-forest 
Table 9. Species of regressive seasonal evergreen formations.

\begin{tabular}{ccc}
\hline Plant species & Abundance & Stratigraphic level \\
\hline Bourreria succulent & ++ & $\mathrm{S} 3 / \mathrm{S} 2$ \\
Citharexylum spinosum & ++++ & $\mathrm{S} 2$ \\
Coccoloba venosa & ++++ & $\mathrm{S} 3$ \\
Diospyros inconstans & +++ & $\mathrm{S} 2$ \\
Jacquinia armillaris & + & $\mathrm{S} 3$ \\
Malpighia emarginata & + & $\mathrm{S} 3$ \\
Randia aculeata & + & $\mathrm{S} 3$ \\
Croton flavens & ++ & $\mathrm{S} 3$ \\
Cordia globosa & + & $\mathrm{S} 3 / \mathrm{S} 2$ \\
Capparis odoratissima & + & $\mathrm{S} 3$ \\
\hline
\end{tabular}

(++++++): very high Abundance/(++++): high Abundance/(+++): medium Abundance/(++): low Abundance/(+): very low Abundance. S1: Upper strata/S2: Middle strata / S3: Lower strata.

stage. The different arrangements formed by the rest of the species show a notable variability of certain factors, including the methods of diaspore dispersal.

With respect to the differences in abundance of the species in Table 10, this plant unit at the pre-forest stage consists of Pisonia fragrans and Leucaena leucocephala. These two tree species emerge from a bush matrix composed of Acacia nilotica and Pithecellobium unguis-cati.

\section{2) R. A. Howard (1950): Kick'Em Jenny}

The species in Table 11(a) correspond to a preforest Phytocenosis mainly composed of Pisonia fragrans and Bursera simaruba which dominate bush units with Capparis odoratissima and Acacia tamarindifolia. Epiphytes typical of dry areas have dense populations: Aechmea lingulata, Epidendrum ciliare, Passiflora suberosa, Cissus verticillata, Tillandsia utriculata and Tillandsia flexuosa.

In this other example, Table 11(b) indicates a preforest grouping dominated by Croton flavens, Cordia curassavica and Pilosocereus royeni from which Coccoloba venosa emerges intermittently.

\section{3) R. A. Howard (1950): Carriacou (the largest island in the Grenadines)}

The plant association in Table 12 dominated by Tabebuia heterophylla and Pisonia fragrans respectively is in the preforest stage. Jacquinia armillaris forms a sparse lower stratum.

\section{4) J. S. BEARD (1949): The Grenadines}

In Table 13, Lonchocarpus violaceus and Swietenia mahagoni (native species to tropical America) are dominant. Haematoxylon campechianum (native to tropical America) often forms a dense, almost impenetrable, lower stratum.

In this phytocenosis of Table 14, two strata are observable. In reality, the vertical distribution of the crowns is heterogeneous and the average values make it possible to define two height classes corresponding to the upper and lower strata. Within this regressive cortege, some species of the advanced stages of 
Table 10. Seasonal evergreen grouping.

\begin{tabular}{ccc}
\hline Plant species & Abundance & Stratigraphic level \\
\hline Acacia nilotica & +++++ & $\mathrm{S} 3 / \mathrm{S} 2$ \\
Celtis iguanea & + & $\mathrm{S} 3$ \\
Leucaena leucocephala & ++ & $\mathrm{S} 3 / \mathrm{S} 2$ \\
Pisonia fragrans & ++++ & $\mathrm{S} 3 / \mathrm{S} 2$ \\
Pithecellobium unguis-cati & ++++++ & $\mathrm{S} 3$ \\
\hline
\end{tabular}

$(++++++)$ : very high Abundance/(++++): high Abundance/(+++): medium Abundance/(++): low Abundance/(+): very low Abundance. S1: Upper strata/S2: Middle strata/S3: Lower strata.

Table 11. (a) Seasonal evergreen associations; (b) Seasonal evergreen associations.

(a)

\begin{tabular}{ccc}
\hline Plant species & Abundance & Stratigraphic level \\
\hline Acacia tamarindifolia & ++++ & $\mathrm{S} 3$ \\
Bursera simaruba & +++ & $\mathrm{S} 2 / \mathrm{S} 1$ \\
Capparis odoratissima & ++++ & $\mathrm{S} 3$ \\
Ficus citrifolia & + & $\mathrm{S} 3$ \\
Pisonia fragrans & ++ & $\mathrm{S} 2 / \mathrm{S} 1$ \\
\hline
\end{tabular}

$(+++++)$ : very strong Abundance/(++++): strong Abundance/(+++): Medium Abundance/(++): weak Abundance/(+): very weak Abundance. S1: Upper strata/S2: Middle strata/S3: Lower strata.

(b)

\begin{tabular}{ccc}
\hline Plant species & Abundance & Stratigraphic level \\
\hline Coccoloba venosa & ++ & $\mathrm{S} 3$ \\
Cordia curassavica & +++ & $\mathrm{S} 3$ \\
Croton flavens & ++++ & $\mathrm{S} 3$ \\
Pilosocereus royeni & +++ & $\mathrm{S} 3$ \\
Senna obtusifolia & + & $\mathrm{S} 3$ \\
Solanum racemosum & + & $\mathrm{S} 3$ \\
\hline
\end{tabular}

$(++++++)$ : very high Abundance/(++++): high/Abundance/(+++): medium Abundance/(++): low Abundance/(+): very low Abundance. S1: Upper strata/S2: Middle strata/S3: Lower strata.

Table 12. Tropical seasonal evergreen young forest of lower horizon.

\begin{tabular}{ccc}
\hline Plant species & Abundance & Stratigraphic level \\
\hline Jacquinia armillaris & ++ & $\mathrm{S} 3$ \\
Pisonia fragrans & ++++ & $\mathrm{S} 2 / \mathrm{S} 1$ \\
Tabebuia heterophylla & ++++++ & $\mathrm{S} 2 / \mathrm{S} 1$ \\
\hline
\end{tabular}

$(++++++)$ : very high Abundance/(++++): high Abundance/(+++): medium Abundance/(++): low Abundance/(+): very low Abundance. S1: Upper strata/S2: Middle strata/S3: Lower strata.

plant succession persist or are installing themselves, such as Brosimum alicastrum, Sideroxylon foetidissimum, Sideroxylon salicifolium, Cordia alliodora, Hymenaea courbaril, Swartzia simplex and Genipa americana. Whether they 
Table 13. Young forest formation.

\begin{tabular}{ccc}
\hline Plant species & Abundance & Stratigraphic level \\
\hline Haematoxylon campechianum & ++++++ & $\mathrm{S} 3 / \mathrm{S} 2$ \\
Lonchocarpus violaceus & ++++ & $\mathrm{S} 2 / \mathrm{S} 1$ \\
Swietenia mahagoni & +++ & $\mathrm{S} 2 / \mathrm{S} 1$ \\
\hline
\end{tabular}

$(++++++)$ : very high Abundance/(++++): high Abundance/(+++): medium Abundance/(++): low Abundance/(+): very low Abundance. S1: Upper strata/S2: Middle strata/S3: Lower strata.

Table 14. Young forest grouping presenting a beginning of structuring.

\begin{tabular}{|c|c|c|c|c|c|}
\hline Plant species & Abundance & $\begin{array}{c}\text { Stratigraphic } \\
\text { level }\end{array}$ & Plant species & Abundance & $\begin{array}{c}\text { Stratigraphic } \\
\text { level }\end{array}$ \\
\hline Albizia caribaea & + & S2 & Ficus citrifolia & + & S2 \\
\hline $\begin{array}{l}\text { Brosimum } \\
\text { alicastrum }\end{array}$ & + & S2/S3 & Genipa americana & + & S2 \\
\hline Bursera simaruba & ++++ & $\mathrm{S} 2 / \mathrm{S} 1$ & Hymenaea courbaril & + & $\mathrm{S} 2 / \mathrm{S} 1$ \\
\hline $\begin{array}{c}\text { Capparis } \\
\text { cynophallophora }\end{array}$ & ++ & $\mathrm{S} 3 / \mathrm{S} 2$ & $\begin{array}{c}\text { Lonchocarpus } \\
\text { violaceus }\end{array}$ & +++ & $\mathrm{S} 2 / \mathrm{S} 1$ \\
\hline Casearia guianensis & ++ & S2 & $\begin{array}{c}\text { Sideroxylon } \\
\text { foetidissimum }\end{array}$ & + & $\mathrm{S} 3 / \mathrm{S} 2$ \\
\hline Cordia alliodora & + & S2/S3 & $\begin{array}{l}\text { Sideroxylon } \\
\text { salicifolium }\end{array}$ & + & S3 \\
\hline $\begin{array}{l}\text { Daphnopsis } \\
\text { americana }\end{array}$ & + & S2 & Swartzia simplex & + & S3 \\
\hline Eugenia monticola & +++ & S3 & $\begin{array}{c}\text { Tabebuia } \\
\text { heterophylla }\end{array}$ & ++++ & $\mathrm{S} 2 / \mathrm{S} 1$ \\
\hline
\end{tabular}

$(+++++)$ : very high Abundance/(++++): high Abundance/(+++): medium Abundance/(++): low Abundance/(+): very low Abundance. S1: Upper strata/S2: Middle strata/S3: Lower strata.

come from an old mature or climactic forest or whether they are in the regeneration phase, these species were affected by very small populations and consequently had no ecological significance at this stage of evolution of the groundcover. Whatever their position in the primitive bipolar structure (matrix/gaps) was, the trees from the final chrono-sequences previously cited are common to the vast majority of the Lesser Antilles. Given the anthropogenic degradations of the time, this spatially very marginal and still structured organisation of the groundcover of Carriacou, the largest and highest islet of the small archipelago of the Grenadines, was a highly degraded example of the old or primary plant ecosystems.

These two examples of predominant old plant associations in the Grenadines reflect the extreme degradation of the original forest floor. The plant groupings belonged to the physiognomic types, bush, shrubland and herbaceous types. The plant communities were all heavily anthropised and derived from the tropical seasonal evergreen sylva of lower horizon in its most xeric facies. Human activities from the beginning and the low rainfall (on average $1000 \mathrm{~mm} / \mathrm{year}$ ) gave the 
landscapes of this archipelago a singularly dry appearance.

\section{5) J. P Fiard, 1990: Union Island}

Autochthonous and naturalised species participating in the various preforest and young forest communities (summary of a set of stations of various minimum areas from $400 \mathrm{~m}^{2}$ to $800 \mathrm{~m}^{2}$ ).

At the time the surveys were carried out, all units were in stages of decline. The phenology of a number of plants and the physiognomies described by the author at the end of the 20th century can be likened to those of semi-deciduous sylva in the tropical dry season, while the physical environment potentially leads to tropical seasonal evergreen groups of lower horizon. The eco-climatic factors of this island give rise to very long climatic droughts (average annual rainfall: $970 \mathrm{~mm}$ ). Naturally, the opening of the forest roof due to land clearing led to an increasing phasing between the intra-vegetation microclimate and the macroclimate, with the appearance of secondary species more tolerant to light (heliophilous) with more general dynamic profiles. Via multiple combinations, the species in Table 15 give rise to young presylvatic and young secondary sylvatic phytocenoses (post-pioneer stages). The latter are dominated (density of individuals and population biomass) by Pisonia fragrans as well as in places with skeletal and rocky soils, Bursera simaruba and Lonchocarpus violaceus. The local eco-climatic conditions allow for the same successive phase, the emergence of mono- or multi-specific stands of Pisonia fragrans (Mount Parnassus), Pisonia fragrans and Lonchocarpus violaceus (Colin Cambelle Reserve).

Still today on this dry (high xericity) and heavily anthropised island, the taxa of Table 15 make up the most advanced forest units which are in the structured secondary dynamic stage. These forest units are characterised by species such as Bursera simaruba, Pisonia fragrans, Lonchocarpus violaceus, Albizzia caribaea, and Spondias mombin (Water Walk Reserve, peaks of Mount Taboï) which create the secondary forest. They form the upper strata and are more abundant than a set of heliophilous trees of lower strata dominated by Bourreria succulenta, Casearia decandra, Guettarda scabra and more rarely Bunchosia glandulosa and Chionanthus compacta. The species mentioned above constituting the lower strata can include all physiognomic types (shrub, bush, tree) and perpetuate themselves in late sylvan organisations as auxiliary species. Within these forest communities, species from the more advanced dynamic stages regenerate in a marginal fashion, e.g. Maytenus laevigta, Genipa americana and Ocotea coriacea, which were probably formerly part of climactic corteges of the primitive vegetation. Although they install from the barely structured preforest or young forest stage thanks to their dynamic profile, they once persisted in the final stages of the plant succession. Due to its mesological conditions, it is likely that Union Island was in the pre-colonial era the best place for the development of the tropical seasonal evergreen forest in its sub-type of lower horizon and in its most xeric facies. In the low islands and within the lower plant stage of the mountainous islands, the architecture and structure of what is known as the pre-Columbian forest depended on very specialised species like Courbaril 
Table 15. Preforest and young forest communities.

\begin{tabular}{|c|c|c|c|c|c|}
\hline Plant species & Abundance & $\begin{array}{c}\text { Stratigraphic } \\
\text { level }\end{array}$ & Plant species & Abundance & $\begin{array}{c}\text { Stratigraphic } \\
\text { level }\end{array}$ \\
\hline Acacia macracantha & + & S3 & Exostema caribaeum & + & $\mathrm{S} 3 / \mathrm{S} 2$ \\
\hline Acacia nilotica & + & S3 & Ficus citrifolia & + & S2 \\
\hline Acacia tamarindifolia & + & S3 & $\begin{array}{c}\text { Forestiera } \\
\text { rhamnifolia }\end{array}$ & + & $\mathrm{S} 2 / \mathrm{S} 3$ \\
\hline Albizzia caribaea & ++ & $\mathrm{S} 2 / \mathrm{S} 1$ & Genipa americana & + & S2 \\
\hline Albizzia lebbeck & + & $\mathrm{S} 1 / \mathrm{S} 2$ & Guettarda scabra & ++++ & $\mathrm{S} 3 / \mathrm{S} 2$ \\
\hline Amyris elemifera & + & S3 & Hymenaea courbaril & + & $\mathrm{S} 2 / \mathrm{S} 1$ \\
\hline $\begin{array}{l}\text { Anacardium } \\
\text { occidendale }\end{array}$ & + & S3 & $\begin{array}{c}\text { Hippomane } \\
\text { mancinella }\end{array}$ & + & $\mathrm{S} 2 / \mathrm{S} 1$ \\
\hline Annona muricata & + & S3 & Jacquinia armillaris & + & S3 \\
\hline Annona squamosa & + & S3 & $\begin{array}{l}\text { Krugiodendron } \\
\text { ferreum }\end{array}$ & + & S2 \\
\hline Bourreria succulenta & ++++++ & S3 & Laetia thamnia & + & $\mathrm{S} 2 / \mathrm{S} 3$ \\
\hline Bunchosia glandulosa & ++ & S3 & $\begin{array}{c}\text { Leucaena } \\
\text { leucocephala }\end{array}$ & + & S3 \\
\hline Bursera simaruba & ++++ & $\mathrm{S} 1 / \mathrm{S} 2$ & $\begin{array}{l}\text { Lonchocarpus } \\
\text { pentaphyllus }\end{array}$ & + & $\mathrm{S} 2 / \mathrm{S} 1$ \\
\hline Caesalpinia coriaria & + & S2 & $\begin{array}{c}\text { Lonchocarpus } \\
\text { violaceus }\end{array}$ & ++++ & $\mathrm{S} 1 / \mathrm{S} 2$ \\
\hline Calotropis procera & + & S3 & $\begin{array}{l}\text { Malpighia } \\
\text { emarginata }\end{array}$ & + & S3 \\
\hline Capparis flexuosa & + & S3 & Margaritaria nobilis & + & $\mathrm{S} 2 / \mathrm{S} 1$ \\
\hline Capparis hastata & + & S3 & Maytenus laevigata & + & $\mathrm{S} 2 / \mathrm{S} 3$ \\
\hline Capparis odoratissima & + & $\mathrm{S} 3$ & Ocotea coriacea & + & $\mathrm{S} 3 / \mathrm{S} 2$ \\
\hline Casearia decandra & ++++ & $\mathrm{S} 2 / \mathrm{S} 3$ & Pisonia fragrans & ++++++ & $\mathrm{S} 2 / \mathrm{S} 1$ \\
\hline Catesbea melanocarpa & + & S3 & $\begin{array}{l}\text { Pithecellobium } \\
\text { unguis-cati }\end{array}$ & + & S3 \\
\hline Ceiba pentandra & + & S1 & Plumeria alba & + & $\mathrm{S} 2 / \mathrm{S} 3$ \\
\hline $\begin{array}{c}\text { Chionanthus } \\
\text { compacta }\end{array}$ & ++ & $\mathrm{S} 2 / \mathrm{S} 3$ & Psychotria microdon & + & S3 \\
\hline $\begin{array}{l}\text { Citharexylum } \\
\text { spinosum }\end{array}$ & + & S2/S3 & Rauvolfia viridis & + & S3 \\
\hline Coccoloba coronata & + & $\mathrm{S} 2 / \mathrm{S} 3$ & $\begin{array}{l}\text { Schaefferia } \\
\text { frutescens }\end{array}$ & + & S3 \\
\hline coccoloba uvifera & + & $\mathrm{S} 2 / \mathrm{S} 3$ & Schoepfia schreberi & + & S3 \\
\hline Comocladia dodonaea & + & S3 & $\begin{array}{l}\text { Sideroxylon } \\
\text { foedissimum }\end{array}$ & + & $\mathrm{S} 2 / \mathrm{S} 3$ \\
\hline Cordia collococca & + & $\mathrm{S} 2 / \mathrm{S} 3$ & Spondias mombin & + & $\mathrm{S} 2 / \mathrm{S} 1$ \\
\hline Cordia obliqua & + & $\mathrm{S} 2 / \mathrm{S} 3$ & $\begin{array}{c}\text { Tabebuia } \\
\text { heterophylla }\end{array}$ & + & $\mathrm{S} 2 / \mathrm{S} 1$ \\
\hline Erithalis odorifera & + & S3 & Tamarindus indica & + & S2 \\
\hline $\begin{array}{c}\text { Erythroxylon } \\
\text { hananense }\end{array}$ & + & S3 & Tecoma stans & + & S3 \\
\hline Eugenia cordata & + & S3 & Trichilia hirta & + & $\mathrm{S} 2 / \mathrm{S} 3$ \\
\hline Eugenia ligustrina & + & S3 & Eugenia rhombea & + & S3 \\
\hline
\end{tabular}

(++++++): very high Abundance/(++++): high Abundance/(+++): medium Abundance/(++): low Abundance/(+): very low Abundance. S1: Upper strata/S2: Middle strata/S3: Lower strata. 
(Hymenaea courbaril) and False Mastic (Sideroxylon foetidissimum). As far as sylvigenesis is concerned, the severe eco-climactic conditions prevailing on this island are limited. Human activities have resulted in the disappearance of the primitive vegetation. Even in sectors that have been exempt from anthropisation for decades, a return to the initial structuring is unlikely. As well as the marked xericity of the biotopes, there is also the erosion of the floristic diversity and the loss of efficiency in the dissemination of seeds.

\subsubsection{J. S. Beard, 1949: Antigua}

Species of tropical seasonal evergreen forest corteges, some of which colonise xeric environments on the lower stage (low islands and certain littoral areas on mountainous islands subject to eco-climatic conditions which lead to long climatic droughts).

1) Wallings Reservoir

In Table 16, the consistent species are, in order of dominance: Inga laurina, Pisonia fragrans, Daphnopsis americana and Mangifera indica. Some taxa of advanced dynamic or climactic phases such as the Courbaril (Hymenaea courbari), the Spanish cedar (Cedrela odorata) and the Spanish elm (Cordia alliodora) regenerate tentatively. However, the latter two (Cordia alliodora, Cedrela odorata) are "scars" of gaps in mature forest formations. Because the species of climactic groupings are absent due to anthropisation, the forest matrix is mainly formed of less specialised secondary species and typical of the windthrow of advanced sylva. This symbolises the acute decline of the original vegetation.

\section{2) Brecknocks Reservoir}

As regards Table 17, Haematoxylon campechianum, Guettarda scabra, Randia aculeata and Exostema caribaeum constitute a dense floristic unit from which some Tabebuia heterophylla emerge whose overall phytomass is much more significant.

\subsubsection{J. S. Beard (1949): Barbuda}

The following species have a dynamic profile which enables them to participate in the successive processes from the start of the tree stages (Table 18). A small number of them persist in the most complex floristic combinations corresponding to the climax: Sideroxylon obovatum, Sideroxylon salicifolium, Pimenta racemosa, Amyris elemifera.

The species in Table 19 form a very dense bush physiological unit within which the first representatives of the young forest stage install and develop. Coccoloba krugii, Byrsonima lucida, Gyminda latifolia and Guettarda scabra are the predominant trees. Taxa, in marginal areas, belonging to a much more septentrional floristic region participate with very little demographic success in certain phytocenoses: Eugenia bahamensis, Eugenia sintensii, Phyllantus angustifolius.

Some examples of associations 
Table 16. Tropical seasonal evergreen formation of lower horizon at structured secondary forest stage.

\begin{tabular}{|c|c|c|c|c|c|}
\hline Plant species & Abundance & $\begin{array}{c}\text { Stratigraphic } \\
\text { level }\end{array}$ & Plant species & Abundance & $\begin{array}{c}\text { Stratigraphic } \\
\text { level }\end{array}$ \\
\hline $\begin{array}{l}\text { Acrocomia } \\
\text { aculeata }\end{array}$ & + & S3 & Hymenaea courbaril & + & S1 \\
\hline Albizzia lebbeck & + & $\mathrm{S} 3 / \mathrm{S} 2$ & Inga laurina & ++++++ & S1 \\
\hline Bucida buceras & ++ & S3 & $\begin{array}{l}\text { Lonchocarpus } \\
\text { pentaphyllus }\end{array}$ & + & S1 \\
\hline Bursera simaruba & + & $\mathrm{S} 3 / \mathrm{S} 2$ & $\begin{array}{l}\text { Lonchocarpus viola- } \\
\text { ceus }\end{array}$ & + & S1 \\
\hline $\begin{array}{l}\text { Byrsonima } \\
\text { spicata }\end{array}$ & + & $\mathrm{S} 3 / \mathrm{S} 2 / \mathrm{S} 1$ & Mangifera indica & +++ & S1 \\
\hline $\begin{array}{c}\text { Casearia } \\
\text { guianensis }\end{array}$ & + & S3 & Melococcus bijugatus & + & S2 \\
\hline Cedrela odorata & + & S1 & Ocotea patens & + & S2 \\
\hline $\begin{array}{c}\text { Ceiba } \\
\left.\text { pentandra }{ }^{*}\right)\end{array}$ & + & S1 & Persea americana & + & S2 \\
\hline $\begin{array}{c}\text { Chionanthus } \\
\text { compacta }\end{array}$ & + & S2 & Pisonia fragrans & +++++ & S1 \\
\hline $\begin{array}{l}\text { Chrysophyllum } \\
\text { argenteum }\end{array}$ & + & S2 & Pisonia subcordata & + & S3 \\
\hline $\begin{array}{l}\text { Coccoloba } \\
\text { pubescens }\end{array}$ & + & S2 & Roystonea oleracea & + & S1 \\
\hline Coccoloba venosa & + & S3 & Sapindus saponaria & + & S2 \\
\hline $\begin{array}{c}\text { Coccothrinax } \\
\text { barbadensis }\end{array}$ & + & S3 & $\begin{array}{l}\text { Sideroxylon } \\
\text { foetidissimum }\end{array}$ & + & S1 \\
\hline Cordia alliodora & + & S1 & Spondias mombin & + & S1 \\
\hline Cordia sulcata & + & S2 & Swietenia mahagoni & + & S1 \\
\hline $\begin{array}{l}\text { Daphnopsis } \\
\text { americana }\end{array}$ & ++++ & S2 & Tabebuia heterophylla & + & S1 \\
\hline Ficus trigonata & + & S1 & $\begin{array}{c}\text { Tabernaemontana } \\
\text { citrifolia }\end{array}$ & + & S2 \\
\hline $\begin{array}{l}\text { Guazuma } \\
\text { ulmifolia }\end{array}$ & + & S2 & $\begin{array}{l}\text { Zanthoxylum } \\
\text { martinicense }\end{array}$ & + & S2 \\
\hline
\end{tabular}

(++++++): very high Abundance/(++++): high Abundance/(+++): medium Abundance/(++): low Abundance/(+): very low Abundance. S1: Upper strata/S2: Middle strata/S3: Lower strata.

- Pure unit of Bucida buceras (++++++)

- Unit of Bucida buceras (+++++), Coccothrinax Barbadensis (++++) and Cactus $s p(+++)$

- Unit of Bursera simaruba (++++), Pisonia fragrans $(+++)$, Canella winterana $(++++)$, Ficus laevigata $(+)$ and Plumeria alba $(++)$

These bush formations and presylvatics or young sylvatics are the various aspects of the regression of the primitive groundcover. However, the characteristics 
Table 17. Extremely regressive form of the seasonal evergreen sylva of lower horizon.

\begin{tabular}{|c|c|c|c|c|c|}
\hline Plant species & Abundance & $\begin{array}{c}\text { Stratigraphic } \\
\text { level }\end{array}$ & Plant species & Abundance & $\begin{array}{c}\text { Stratigraphic } \\
\text { level }\end{array}$ \\
\hline $\begin{array}{c}\text { Aegiphila } \\
\text { martinicensis }\end{array}$ & + & S3 & $\begin{array}{l}\text { Haematoxylon } \\
\text { campechianum }\end{array}$ & ++++++ & S3 \\
\hline Albizia lebbeck & + & S2 & Lantana involucrata & + & S3 \\
\hline Amphitecna latifolia & + & S3 & $\begin{array}{c}\text { Leucaena } \\
\text { leucocephala }\end{array}$ & + & S3 \\
\hline Bourreria succulenta & + & S3 & Melochia tomentosa & + & $?$ \\
\hline $\begin{array}{c}\text { Capparis } \\
\text { cynophallophora }\end{array}$ & + & S3 & Myrcia citrifolia & + & S3 \\
\hline Capparis indica & + & S3 & $\begin{array}{c}\text { Piscidia } \\
\text { carthagenensis }\end{array}$ & + & S3 \\
\hline Casearia guianensis & + & S3 & Pisonia $s p$ & + & $?$ \\
\hline $\begin{array}{c}\text { Chamaecrista } \\
\text { gladulosa var.swartzii }\end{array}$ & + & S3 & Prosopis juliflora & + & S2 \\
\hline $\begin{array}{l}\text { Chrysophyllum } \\
\text { argenteum }\end{array}$ & + & S2 & Psidium guajava & + & S3 \\
\hline $\begin{array}{l}\text { Citharexylum } \\
\text { spinosum }\end{array}$ & + & S2 & Randia aculeata & +++++ & S3 \\
\hline Comocladia dodonaea & + & S3 & $\begin{array}{c}\text { Solanum } \\
\text { racemosum }\end{array}$ & + & S3 \\
\hline Croton flavens & + & S3 & Swietenia mahagoni & + & S1 \\
\hline $\begin{array}{l}\text { Daphnopsis } \\
\text { americana }\end{array}$ & + & S2 & $\begin{array}{c}\text { Tabebuia } \\
\text { heterophylla }\end{array}$ & + & S1 \\
\hline $\begin{array}{c}\text { Erythroxylon } \\
\text { havanense }\end{array}$ & + & S3 & Tecoma stans & + & S3 \\
\hline Exostea caribaeum & +++ & S2 & $\begin{array}{c}\text { Tetrazygia } \\
\text { angustifolia }\end{array}$ & + & S3 \\
\hline Guettarda scabra & ++++++ & S2 & $\begin{array}{l}\text { Zanthoxylum } \\
\text { monophyllum }\end{array}$ & + & S3 \\
\hline
\end{tabular}

$(++++++)$ : very high Abundance/(++++): high Abundance/(+++): medium Abundance/(++): low Abundance/(+): very low Abundance. S1: Upper strata/S2: Middle strata/S3: Lower strata.

Table 18. Cortege of species from secondary tropical seasonal evergreen forest islets.

\begin{tabular}{|c|c|c|c|c|c|}
\hline Plant species & Abundance & $\begin{array}{c}\text { Stratigraphic } \\
\text { level }\end{array}$ & Plant species & Abundance & $\begin{array}{c}\text { Stratigraphic } \\
\text { level }\end{array}$ \\
\hline Amyris elemifera & + & S3 & Pimenta racemosa & ++ & SI/S2 \\
\hline Bucida buceras & ++ & $\mathrm{S} 3 / \mathrm{S} 2$ & Pisonia fragrans & ++ & $\mathrm{S} 1 / \mathrm{S} 2 / \mathrm{S} 3$ \\
\hline Bursera simaruba & ++++ & $\mathrm{S} 2 / \mathrm{S} 1$ & Pisonia subcordata & + & S3 \\
\hline Canella winterana & ++ & $\mathrm{S} 3 / \mathrm{S} 2$ & $\begin{array}{c}\text { Sideroxylon } \\
\text { obovatum }\end{array}$ & + & $\mathrm{S} 1 / \mathrm{S} 2 / \mathrm{S} 3$ \\
\hline $\begin{array}{l}\text { Coccoloba } \\
\text { diversifolia }\end{array}$ & +++ & $\mathrm{S} 2 / \mathrm{S} 3$ & $\begin{array}{l}\text { Sideroxylon } \\
\text { salicifolium }\end{array}$ & + & $\mathrm{S} 1 / \mathrm{S} 2$ \\
\hline Ficus citrifolia & + & S2 & $\begin{array}{c}\text { Tabebuia } \\
\text { heterophylla }\end{array}$ & ++++ & $\mathrm{S} 1 / \mathrm{S} 2 / \mathrm{S} 3$ \\
\hline $\begin{array}{l}\text { Lonchocarpus } \\
\text { pentaphyllus }\end{array}$ & +++ & $\mathrm{S} 2 / \mathrm{S} 2 / \mathrm{S} 1$ & Zanthoxylum flavum & ++ & $\mathrm{S} 1 / \mathrm{S} 2 / \mathrm{S} 3$ \\
\hline
\end{tabular}

(++++++): very high Abundance/(++++): high Abundance/(+++): medium Abundance/(++): low Abundance/(+): very low Abundance. S1: Upper strata/S2: Middle strata/S3: Lower strata. 
Table 19. Species of presylvatic regressive plant associations.

\begin{tabular}{|c|c|c|c|c|c|}
\hline Plant species & Abundance & $\begin{array}{c}\text { Stratigraphic } \\
\text { level }\end{array}$ & Plant species & Abundance & $\begin{array}{c}\text { Stratigraphic } \\
\text { level }\end{array}$ \\
\hline Bourreria succulenta & +++ & $\mathrm{S} 2 / \mathrm{S} 3$ & Eugenia cordata & + & S3 \\
\hline Byrsonima lucida & ++++ & $\mathrm{S} 2 / \mathrm{S} 3$ & Guettarda scabra & ++++ & $\mathrm{S} 2 / \mathrm{S} 3$ \\
\hline $\begin{array}{c}\text { Capparis } \\
\text { cynophallophora }\end{array}$ & + & S3 & Gyminda latifolia & ++++ & $\mathrm{S} 2 / \mathrm{S} 1$ \\
\hline Coccoloba krugii & ++++ & S2 & $\begin{array}{l}\text { Jacquinia } \\
\text { armillaris }\end{array}$ & + & S3 \\
\hline $\begin{array}{c}\text { Coccothrinax } \\
\text { barbadensis }\end{array}$ & ++ & $\mathrm{S} 2 / \mathrm{S} 3$ & Malpighia linearis & + & S3 \\
\hline Comocladia dodonaea & + & S3 & $\begin{array}{c}\text { Phyllanthus } \\
\text { (angustifolius)? }\end{array}$ & + & S3 \\
\hline Dodonaea viscosa & + & S3 & $\begin{array}{l}\text { Pithecellobium } \\
\text { unguis-cati }\end{array}$ & ++ & S3 \\
\hline Eugenia axillaris & ++ & S3 & Plumeria alba & + & $\mathrm{S} 3 / \mathrm{S} 2$ \\
\hline Eugenia (Bahamensis)? & + & S3 & & & \\
\hline
\end{tabular}

$(++++++)$ : very high Abundance/(++++): high Abundance/(+++): medium Abundance/(++): low Abundance/(+): very low Abundance. S1: Upper strata/S2: Middle strata/S3: Lower strata.

of the macroclimate are scarcely required for the installation of a forest cover. Secondary plant units are similar to hyper-xerophytic vegetation.

\subsubsection{J. S. Beard (1949): Barbados}

Table 20 presents a floristic grouping corresponding to the secondary tropical seasonal evergreen sylva in its sub-type of lower horizon, in which species of advanced dynamic stages find the potential for installation and probable expansion: Sideroxylon salicifolium, Manilkara bidentata for the most specialised.

\subsubsection{H. Stehle (1937): Barbados}

This survey by H. Stehle in Barbados (north centre) in 1937, summarised in Table 21, bears certain resemblances to the previous survey carried out on the same island, in 1945, by J.S. Beard (Table 20). In terms of ecosystemic analysis, we can formulate identical conclusions. Namely, that the inventoried floristic composition and the architectural organisation described refer to the following forest type: tropical seasonal evergreen sylva, in its sub-type of lower horizon and in an intra-sylvatic regressive dynamic facies (secondary structured). The post-pioneer sylvan species are dominant: in order of significance, Lonchocarpus violaceus, Bursera simaruba and Coccoloba pubescens.

\subsubsection{The French Antilles}

In Martinique and Guadeloupe, we offer, in illustration, some aspects of the groundcover, in the form of predominant floristic compositions.

1) Martinique (J. P. Fiard, 1992): Terre Rouge (peak, altitude 280 m, survey of $950 \mathrm{~m}^{2}$ ) 
Table 20. Secondary tropical seasonal evergreen sylva.

\begin{tabular}{|c|c|c|c|c|c|}
\hline Plant species & Abundance & $\begin{array}{c}\text { Stratigraphic } \\
\text { level }\end{array}$ & Plant species & Abundance & $\begin{array}{c}\text { Stratigraphic } \\
\text { level }\end{array}$ \\
\hline Aiphanes erosa & + & $\mathrm{S} 2 / \mathrm{S} 3 / \mathrm{S} 1$ & Hernendia sonora & + & $\mathrm{S} 2 / \mathrm{S} 3$ \\
\hline $\begin{array}{l}\text { Bunchosia } \\
\text { glandulosa }\end{array}$ & + & S3 & Hura crepitans & + & $\mathrm{S} 1 / \mathrm{S} 2$ \\
\hline Bursera simaruba & +++ & $\mathrm{S} 1 / \mathrm{S} 2$ & Hymenaea courbaril & + & $\mathrm{S} 1 / \mathrm{S} 2$ \\
\hline Byrsonima spicata & +++ & $\mathrm{S} 2 / \mathrm{S} 1$ & Inga laurina & + & $\mathrm{S} 1 / \mathrm{S} 2$ \\
\hline Casearia decandra & ++ & S3 & Maclura tinctoria & + & $\mathrm{S} 2 / \mathrm{S} 3$ \\
\hline Cedrela odorata & ++ & $\mathrm{S} 1 / \mathrm{S} 2$ & Manilkara bidentata & + & $\mathrm{S} 1 / \mathrm{S} 2$ \\
\hline Ceiba pentandra & + & S1 & Ocotea patens & + & $\mathrm{S} 2 / \mathrm{S} 3$ \\
\hline $\begin{array}{c}\text { Citharexylum } \\
\text { spinosum }\end{array}$ & + & S3 & Pisonia fragrans & ++ & $\mathrm{S} 2 / \mathrm{S} 3$ \\
\hline $\begin{array}{l}\text { Coccoloba } \\
\text { diversifolia }\end{array}$ & ++ & $\mathrm{S} 2 / \mathrm{S} 1$ & Roystonea oleacea & ++ & $\mathrm{S} 1 / \mathrm{S} 2$ \\
\hline $\begin{array}{l}\text { Coccoloba } \\
\text { pubescens }\end{array}$ & ++ & $\mathrm{S} 2 / \mathrm{S} 1$ & Sapium Glandulosum & ++ & S3 \\
\hline Coccoloba venosa & + & $\mathrm{S} 3 / \mathrm{S} 2$ & $\begin{array}{l}\text { Sideroxylon } \\
\text { salicifolium }\end{array}$ & + & S3 \\
\hline Cordia collococca & + & $\mathrm{S} 3 / \mathrm{S} 2$ & Simaruba amara & + & $\mathrm{S} 1 / \mathrm{S} 2$ \\
\hline Cordia sulcata & + & $\mathrm{S} 1 / \mathrm{S} 2$ & Tabebuia heterophylla & +++ & $\mathrm{S} 1 / \mathrm{S} 2$ \\
\hline Crateva tapia & + & $\mathrm{S} 2 / \mathrm{S} 3$ & $\begin{array}{l}\text { Zanthoxylum } \\
\text { martinicense }\end{array}$ & + & $\mathrm{S} 2 / \mathrm{S} 3$ \\
\hline Cupania americana & + & $\mathrm{S} 2 / \mathrm{S} 3$ & $\begin{array}{c}\text { Zanthoxylum } \\
\text { punctatum }\end{array}$ & + & S3 \\
\hline $\begin{array}{c}\text { Faramea } \\
\text { occidentalis }\end{array}$ & + & S3 & & & \\
\hline
\end{tabular}

$(++++++)$ : very high Abundance/(++++): high Abundance/(+++): medium Abundance/(++): low Abundance/(+): very low Abundance. S1: Upper stratum/S2: Middle stratum/S3: Lower stratum.

Table 22 shows that the most abundant species are heliophilous and are balanced in the chrono-sequence of succession considered, namely the structured secondary sylvatic stage: Tabebuia heterophylla, Coccoloba swartzii and Eugenia confusa. These three species can appear from the pre-sylvatic and sometimes mature bush stage.

2) Martinique (J. P. Fiard, 1992): Terre Rouge (northern slope, altitude $150-160 \mathrm{~m}$, survey $1000 \mathrm{~m}^{2}$ )

The predominant species in Table 23 are mainly heliophilous and they can be primary (pioneers) or secondary (leading post-pioneers) and show that the "secondary forest" dynamic stage is expanding. In other words, the intra-forest ecological conditions allow the ecologically dominant species to find sites for installation and expansion. The floristic composition found indicates the dry bioclimate. However, the presence in this station of species of minor ecological significance belonging to the tropical seasonal evergreen sylva type and tropical 
Table 21. Tropical seasonal evergreen sylva.

\begin{tabular}{|c|c|c|c|c|c|}
\hline Plant species & Abundance & $\begin{array}{c}\text { Stratigraphic } \\
\text { level }\end{array}$ & Plant species & Abundance & $\begin{array}{l}\text { Stratigraphic } \\
\text { level }\end{array}$ \\
\hline $\begin{array}{l}\text { Aiphanes minima } \\
\text { (Aiphanes erosa?) }\end{array}$ & + & S3 & Hura crepitans & + & $\mathrm{S} 1 / \mathrm{S} 2$ \\
\hline Bursera simaruba & +++ & $\mathrm{S} 1 / \mathrm{S} 2$ & $\begin{array}{c}\text { Hymenaea } \\
\text { courbaril }\end{array}$ & + & S1./S2 \\
\hline $\begin{array}{c}\text { Cecropia } \\
\text { schreberiana }\end{array}$ & + & S1 & $\begin{array}{c}\text { Lonchocarpus } \\
\text { violaceus }\end{array}$ & ++++ & $\mathrm{S} 1 / \mathrm{S} 2$ \\
\hline Cedrela odorata & + & $\mathrm{S} 1 / \mathrm{S} 2$ & Maclura tinctoria & + & $\mathrm{S} 2 / \mathrm{S} 3$ \\
\hline Ceiba pentandra & + & S1 & $\begin{array}{c}\text { Roystenia regia } \\
\text { (Roystenia olea- } \\
\text { cea?) }\end{array}$ & + & $\mathrm{S} 1 / \mathrm{S} 2$ \\
\hline $\begin{array}{l}\text { Citharexylum spi- } \\
\text { nosum }\end{array}$ & ++ & S3 & $\begin{array}{c}\text { Sapium } \\
\text { Hippomane }\end{array}$ & + & $\mathrm{S} 1 / \mathrm{S} 2 / \mathrm{S} 3$ \\
\hline $\begin{array}{l}\text { Coccoloba } \\
\text { pubescens }\end{array}$ & +++ & $\mathrm{S} 1 / \mathrm{S} 2$ & $\begin{array}{l}\text { Sideroxylon } \\
\text { salicifolium }\end{array}$ & + & S2/S3 \\
\hline Cordia glabra? & + & $\mathrm{S} 2 / \mathrm{S} 3$ & $\begin{array}{c}\text { Zanthoxylum } \\
\text { aribaea }\end{array}$ & ++ & $\mathrm{S} 2 / \mathrm{S} 1$ \\
\hline Hernandia sonora & + & $\mathrm{S} 2 / \mathrm{S} 3$ & & & \\
\hline
\end{tabular}

$(++++++)$ : very high Abundance/(++++): high Abundance/(+++): medium Abundance/(++): low Abundance/(+): very low Abundance. S1: Upper stratum/S2: Middle stratum/S3: Lower stratum.

Table 22. Tropical seasonal evergreen forest in maturity phase, in its lower horizon subtype and in its structured secondary dynamic facies.

\begin{tabular}{|c|c|c|c|c|c|}
\hline Plant species & Abundance & $\begin{array}{c}\text { Stratigraphic } \\
\text { levels }\end{array}$ & Plant species & Abundance & $\begin{array}{c}\text { Stratigraphic } \\
\text { levels }\end{array}$ \\
\hline $\begin{array}{l}\text { Bourreria } \\
\text { succulenta }\end{array}$ & ++ & $\mathrm{S} 2 / \mathrm{S} 3$ & Eugenia confusa & ++++++ & S3 \\
\hline Busera simaruba & +++ & $\mathrm{S} 1 / \mathrm{S} 2$ & $\begin{array}{c}\text { Eugenia } \\
\text { lambertiana }\end{array}$ & ++ & S3 \\
\hline Capparis flexuosa & + & S3 & $\begin{array}{c}\text { Eugenia } \\
\text { pseudopsidium }\end{array}$ & ++ & $\mathrm{S} 2 / \mathrm{S} 3$ \\
\hline Casearia decandra & ++ & S3 & $\begin{array}{l}\text { Homalium } \\
\text { racemosum }\end{array}$ & +++ & $\mathrm{S} 1 / \mathrm{S} 2$ \\
\hline $\begin{array}{l}\text { Cassipourea } \\
\text { guianensis }\end{array}$ & +++ & S3 & Inga laurina & ++ & $\mathrm{S} 1 / \mathrm{S} 2$ \\
\hline $\begin{array}{c}\text { Chionanthus } \\
\text { compacta }\end{array}$ & ++ & S3 & Ixora ferrea & + & S3 \\
\hline $\begin{array}{l}\text { Chrysophyllum } \\
\text { argenteum }\end{array}$ & + & S3 & $\begin{array}{c}\text { Lonchocarpus } \\
\text { violaceus }\end{array}$ & +++ & $\mathrm{S} 1 / \mathrm{S} 2$ \\
\hline Clusia major & + & S3 & $\begin{array}{c}\text { Myrcia citrifolia } \\
\text { var. imrayana }\end{array}$ & + & S3 \\
\hline Coccoloba swartzii & ++++++ & $\mathrm{S} 2 / \mathrm{S} 3$ & $\begin{array}{l}\text { Myrciaria } \\
\text { floribunba }\end{array}$ & +++ & S3 \\
\hline $\begin{array}{c}\text { Cordia } \\
\text { martinicensis }\end{array}$ & + & S3 & Pisonia fragrans & +++ & $\mathrm{S} 1 / \mathrm{S} 2 / \mathrm{S} 3$ \\
\hline $\begin{array}{c}\text { Daphnopsis } \\
\text { americana }\end{array}$ & ++ & S3 & Randia aculeata & + & S3 \\
\hline $\begin{array}{c}\text { Erythroxylon } \\
\text { havanense }\end{array}$ & + & S3 & $\begin{array}{c}\text { Tabebuia } \\
\text { hétérophylla }\end{array}$ & ++++++ & $\mathrm{S} 1 / \mathrm{S} 2$ \\
\hline
\end{tabular}

$(++++++)$ : very high Abundance/(++++): high Abundance/(+++): medium Abundance/(++): low Abundance/(+): very low Abundance. S1: Upper stratum/S2: Middle stratum/S3: Lower stratum. 
Table 23. Plant cover consisting of a tropical seasonal evergreen tree formation in its lower horizon subtype and in its secondary dynamic stage.

\begin{tabular}{|c|c|c|c|c|c|}
\hline Plant species & Abundance & $\begin{array}{l}\text { Stratigraphic } \\
\text { level }\end{array}$ & Plant species & Abundance & $\begin{array}{l}\text { Stratigraphic } \\
\text { level }\end{array}$ \\
\hline $\begin{array}{l}\text { Bourreria } \\
\text { succulenta }\end{array}$ & + & S3 & Eugenia ligustrina & + & S3 \\
\hline $\begin{array}{l}\text { Bunchosia } \\
\text { glandulosa }\end{array}$ & + & S3 & Ficus citrifolia & + & S2 \\
\hline Byrsonima spicata & + & $\mathrm{S} 2 / \mathrm{S} 3$ & Inga laurina & + & $\mathrm{S} 2 / \mathrm{S} 3$ \\
\hline Calophyllum calaba & + & $\mathrm{S} 2 / \mathrm{S} 3$ & $\begin{array}{c}\text { Lonchocarpus } v \\
\text { iolaceus }\end{array}$ & ++++++ & $\mathrm{S} 1 / \mathrm{S} 2$ \\
\hline Casearia decandra & + & S3 & $\begin{array}{c}\text { Myrcia citrifolia var } \\
\text { imrayana }\end{array}$ & + & S3 \\
\hline $\begin{array}{l}\text { Cassipourea } \\
\text { guianensis }\end{array}$ & + & S3 & Ocotea cernua & +++ & $\mathrm{S} 2 / \mathrm{S} 3$ \\
\hline $\begin{array}{l}\text { Chrysophyllum } \\
\text { argenteum }\end{array}$ & + & S3 & Ocotea leucoxylon & + & $\mathrm{S} 1 / \mathrm{S} 2$ \\
\hline $\begin{array}{l}\text { Cinnamomum } \\
\text { elongatum }\end{array}$ & + & $\mathrm{S} 3 / \mathrm{S} 2$ & Pisonia fragrans & ++++ & $\mathrm{S} 1 / \mathrm{S} 2$ \\
\hline $\begin{array}{l}\text { Citharexylum } \\
\text { spinosum }\end{array}$ & + & $\mathrm{S} 2 / \mathrm{S} 3$ & Tabebuia heterophylla & ++ & $\mathrm{S} 1 / \mathrm{S} 3$ \\
\hline Coccoloba swartzii & + & $\mathrm{S} 2 / \mathrm{S} 3$ & $\begin{array}{c}\text { Tabernaemontana } \\
\text { citrifolia }\end{array}$ & + & S3 \\
\hline $\begin{array}{l}\text { Conostegia } \\
\text { calyptrata }\end{array}$ & + & S3 & $\begin{array}{c}\text { Zanthoxylum } \\
\text { caribaeum }\end{array}$ & + & $\mathrm{S} 2 / \mathrm{S} 3$ \\
\hline \multirow[t]{2}{*}{ Eugenia gregii } & + & S3 & Sapiumcaribaeum & + & $\mathrm{S} 1 / \mathrm{S} 2$ \\
\hline & & & Simaruba amara & + & $\mathrm{S} 1 / \mathrm{S} 2$ \\
\hline
\end{tabular}

(++++++): very high Abundance/(++++): high Abundance/(+++): medium Abundance/(++): low Abundance/(+): very low Abundance. S1: Upper stratum/S2: Middle stratum/S3: Lower stratum.

seasonal umbro-evergreen sylva, attests to the less xeric nature of this biotope. This is compared to that which prevails in the region near the coast. These species are respectively, Ocotea cernua, Eugenia pseudopsidium, Tabernaemontana citrifolia, Cinnamomum elongatum for the secondary stage and Cassipourea guianensis, Ocotea leucoxylon, Calophyllum calaba for the final stage. It is logical to think that this formation will continue to expand. In the medium term, the resulting formation will be mature for this stage; however, the current predominant species will no longer be balanced and will only constitute its understructure. Concomitantly, the ecologically more specialised species already installed (Ocotea leucoxylon), as well as those originating from the advective potential, will find environmental conditions which are more favourable to their development.

3) Martinique (J. P. Fiard, 1992): Piton Pierreux sector (western ridge, southern slope, altitude $180 \mathrm{~m}, 850 \mathrm{~m}^{2}$ )

This forest community represented in Table 24 is scarcely structured (insignificant stratification). Regenerations of more specialised species from the advanced secondary forest stage are non-existent ${ }^{1}$. For the entire plot, the

${ }^{1}$ The stage above the stage indicated by the population abundance in Table 24 and which is the young forest stage. 
Table 24. Tropical seasonal evergreen sylvatic phytocenosis in its lower horizon subtype and in its structured young secondary dynamic facies.

\begin{tabular}{|c|c|c|c|c|c|}
\hline Plant species & Abundance & $\begin{array}{c}\text { Stratigraphic } \\
\text { level }\end{array}$ & Plant species & Abundance & $\begin{array}{c}\text { Stratigraphic } \\
\quad \text { level }\end{array}$ \\
\hline $\begin{array}{l}\text { Bourreria } \\
\text { succulenta }\end{array}$ & + & S1 & $\begin{array}{l}\text { Lonchocarpus viola- } \\
\text { ceus }\end{array}$ & ++++++ & $\mathrm{S} 1 / \mathrm{S} 2$ \\
\hline Bursera simaruba & +++ & $\mathrm{S} 1 / \mathrm{S} 2$ & $\begin{array}{c}\text { Myrcia citrifolia } \\
\text { var.imrayana }\end{array}$ & + & S3 \\
\hline $\begin{array}{l}\text { Byrsonima } \\
\text { spicata }\end{array}$ & + & $\mathrm{S} 1 / \mathrm{S} 2$ & Picramnia pentandra & + & S3 \\
\hline $\begin{array}{l}\text { Casearia } \\
\text { decandra }\end{array}$ & + & S3 & Pisonia fragrans & +++ & $\mathrm{S} 1 / \mathrm{S} 2$ \\
\hline $\begin{array}{c}\text { Chionanthus } \\
\text { compacta }\end{array}$ & + & S3 & Psychotria microdon & + & S3 \\
\hline $\begin{array}{l}\text { Chrysophyllum } \\
\text { argenteum }\end{array}$ & + & S3 & Randia aculeata & + & S3 \\
\hline $\begin{array}{c}\text { Coccoloba swart- } \\
\text { zii }\end{array}$ & ++++ & $\mathrm{S} 1 / \mathrm{S} 2$ & Tabebuia heterophylla & + & $\mathrm{SS} 2 / \mathrm{S} 3 / \mathrm{S} 1$ \\
\hline $\begin{array}{l}\text { Eugenia } \\
\text { monticola }\end{array}$ & + & S3 & $\begin{array}{c}\text { Tabernaemontana } \\
\text { citrifolia }\end{array}$ & + & S3 \\
\hline $\begin{array}{c}\text { Eugenia } \\
\text { pseudopsidium }\end{array}$ & + & $\mathrm{S} 2 / \mathrm{S} 3$ & $\begin{array}{l}\text { Zanthoxylum } \\
\text { monophyllum }\end{array}$ & + & S3 \\
\hline
\end{tabular}

$(++++++)$ : very high Abundance/(++++): high Abundance/(+++): medium Abundance/(++): low Abundance/(+): very low Abundance. S1: Upper stratum/S2: Middle stratum/S3: Lower stratum.

considerable ecological dominance of edifying species, pioneer heliophilous species and post-pioneer species transitorily balanced from a population point of view $^{2}$, indicates a relatively open state of this forest vegetation. The ecological profile with a xeric tendency of the various auxiliary or regenerating species, definitively shows that here the ecosystemic potentiality is the tropical seasonal evergreen sylva of lower horizon. In the previous survey, Table 23, Pisonia fragrans and Tabebuia heterophylla, species from regressive forest environments, are associated with other edifying species from more humid areas (Sapium caribaeum, Simaruba amara) and sometimes more advanced dynamic stages (Ocotea leucoxylon). The presence of Pisonia fragrans in the dominant cortege, at two different altitudes (Piton Pierreux and Terre Rouge) distinctly corresponding to the medium (humid subhumid) and lower (dry subhumid) bioclimatic stages, in no way shows an analogy of sylvatic potential of the two floristic survey zones. It results from intra-vegetation changes to the ecoclimatic conditions of the phytocenosis of the middle stage. Indeed, the regression of the climactic sylvan groundcover on this stage resulted in the transition from a closed canopy with overlapping crowns to a sparse open canopy with non-contiguous sympodiums. The result is a greater phasing between the intra-vegetation microclimate and the macroclimate, which has given rise to installation and expansion sites specific to some species of the lower stage with broad ecological valency. This is

\footnotetext{
${ }^{2}$ In a balanced population of balanced plant species all age groups are represented.
} 
an illustration of the phenomenon of floristic convergence between two distinct bioclimatic stages. Structural transformations linked to humans or to the effects of natural hazards in the middle plant stage influenced by the humid subhumid bioclimate can reveal floristic similarities with the lower plant stage subjected to the dry subhumid bioclimate.

\section{4) Guadeloupe ${ }^{3}$ (H. Stehle, 1939): The xeric littoral}

The littoral areas, especially those which are leeward (in Basse-Terre), attest as in most of the other islands to a very marked xericity. In fact, difficult climatic conditions are characterised by low rainfall, very high evaporation, cloudiness much lower than in the other bioclimatic areas (especially in dry season, which can be very long) and intense sunshine.

These eco-climatic conditions lead to a pedogenesis which resulted in soils from vertisolisation ${ }^{4}$ and a regressive xerophytic vegetation endowed with very specific morphological (xeromorphosis or ecomorphosis), anatomical and physiological adaptation mechanisms. These adaptations translate in practice into the establishment of water reserves in the tissues (transformation of support bodies) or the reduction to basal metabolism, of water losses by means of evapotranspiration (fluids in specialised cells, cuticles, hairs, etc.).

Despite the xeromorphoses, in its climactic phase the vegetation of the xeric littoral of Basse-Terre in Guadeloupe is sylvatic. What is indicated by data from digital surveys and observations is simply the result of the anthropogenic regression of the primitive forest units. The edifying species are probably very different from the autoecological point of view (temperament in relation to physical factors), than those of the interior spaces of this lower plant stage. In what follows, we will present some examples of sylvatic plant associations which convey floristic particularities among the plurality of potential arrangements influenced by strict mesological and anthropological factors.

\section{5) Guadeloupe (H. Stehle, 1939): Inland regions}

The particular conditions of the coastal regions, in this geographical area, are greatly attenuated and the plant type is dictated as much by eco-climatic as anthropogenic factors.

\section{a) The calcareous plateaus and calcareous hills}

The Grande-Terre of Guadeloupe, Désirade and Marie-Galante are mainly covered with a calcareous substratum dating from the Miocene (around 26 million years: a time when the angiosperms were expanding). The ecosystems of these relatively low regions, from which rise small massifs called mornes, have undergone an anthropisation not always similar in form, but extremely pronounced. Indeed, they were the site for the development of cultures of allochthonous plants of all kinds. This state of affairs has resulted in a regression of the original groundcover. By the time H. Stehle made his observations, the sylva had

${ }^{3}$ To demonstrate in a non-exhaustive fashion the diversity of the forest formations of Guadeloupe and its dependencies, we will use as a framework the reinterpreted descriptions and floristic surveys of H. Stehle (1936), as well as more recent data from our inventories in these dependencies.

${ }^{4}$ Vertisolisation is the process that leads to the formation of vertisols in climates with contrasting seasons, of the subtropical or tropical type. 
decreased considerably in area. And the few forest examples that remained formed marginal islets inserted here and there in a matrix of bush species or in the agricultural flora.

The low altitude of these calcareous islands in relation to the significantly reduced rainfall, the minimal nebulosity and the high evaporation are all mesological factors which lead to a general climate which is highly xeric. The drought period is variable but nevertheless very long. As stated previously, the vegetation of 1936 in these calcareous environments, whatever its physiognomic type and its ecosystemic complexity, derived from that of pre-Columbian times which was essentially sylvan. The constituent units of the latter had reached a high degree of complexity and gave rise to an intra-forest environment with a certain autonomy with regard to the general climate. During the prehistory of the Guadeloupe archipelago, the flora which was predominant was composed of highly specialised species typical of final stages (climax stages). They showed no adaptation to drought (morphological or anatomical). By 1936, the original diversity had been greatly eroded. The different physiognomic units listed result from anthropogenic degradations. Stehle identifies several types of phytocenoses, however the presylvatics constitute a significant component of the landscape.

Grande-Terre (H. Stehle, 1936)

In Table 25, Erythroxylon havanense, Zanthoxylum caribaeum, Zanthoxylum martinicense, Sideroxylon salicifolium, Tabebuia heterophylla and Pisonia fragrans are well represented. These species, depending on the local eco-climatic conditions, can combine in variable ratios of dominance. They form plant units with mature bush or presylvatic physiognomy. The hills of Grande-Terre show an accentuated xericity, the association Sideroxylon obovatum-Erythroxylon havanense is frequently encountered. Indeed, a calciphilous tree species (Sideroxylon obovatum), arriving very early in the dynamic and participating in the advanced stages, is associated with a non-calciphilous pioneer bush species (Erythroxylon havanense) from xeric environments and belonging to the secondary regressive dynamic stages. This formation, composed of a low plant matrix from which the crowns of Sideroxylon obovatum emerge, is the result of an organisational peculiarity of the groundcover, reflecting both very dry bioclimatic conditions and the anthropisation of Grande-Terre. As well as these predominant plants, there are also other trees of lower ecological importance (Table 25).

\section{Marie-Galante (H. Stehle, 1936)}

The units that formed the vegetation all emerged in distinct proportions, from the predominant group of the following species, similar to that found on Grande-Terre in Guadeloupe: Pisonia subcordata, Tabebuia heterophylla, Crossopetalum rhacoma, Byrsonima lucida, Sideroxylon salicifolium, Erythroxylon havanense, Eugenia axillaris and Gossipium barbadense. The latter two species are predominant at the time. However, in certain places a vegetation was found which differed from that mentioned above, by way of a somewhat increased structuring (forest of Folle-Anse (1936)). The main formations mentioned were 
Table 25. Tropical seasonal evergreen sylva.

\begin{tabular}{ccc}
\hline Plant species & Abundance & Stratigraphic level \\
\hline Bucida buceras & ++ & $\mathrm{S} 3 / \mathrm{S} 2$ \\
Byrsonima lucida & ++ & $\mathrm{S} 3 / \mathrm{S} 2$ \\
Coccothrinax barbadensis & + & $\mathrm{S} 3 / \mathrm{S} 2$ \\
Crossopetalum rhacoma & + & $\mathrm{S} 3$ \\
Erythroxylon havanense & ++++++ & $\mathrm{S} 3 / \mathrm{S} 2$ \\
Morinda citrifolia $\left({ }^{*}\right)$ & + & $\mathrm{S} 3$ \\
Pisonia subcordata & ++ & $\mathrm{S} 3$ \\
Sideroxylon obovatum & +++++ & $\mathrm{S} 1 / \mathrm{S} 2$ \\
Tabebuia heterophylla & ++ & $\mathrm{S} 1 / \mathrm{S} 2$ \\
Zanthoxylum caribaeum & + & $\mathrm{S} 2 / \mathrm{S} 3 / \mathrm{S} 1$ \\
Zanthoxylum martinicense & + & $\mathrm{S} 2 / \mathrm{S} 3 / \mathrm{S} 1$
\end{tabular}

$(++++++)$ : very high Abundance/(++++): high Abundance/(+++): medium Abundance/(++): low Abundance/(+): very low Abundance. S1: Upper stratum/S2: Middle stratum/S3: Lower stratum.

partly composed of more specialised species such as, Zanthoxylum punctatum, Zanthoxylum flavum, Exostema sanctae-luciae and Xylosma buxifolium.

La Désirade (H. Stehle, 1936)

According to Table 26, the plateau, the mornes and the west coast hosted a typical flora derived from pre-colonial sylvicultural ecosystems, which was dominated by the association Canella winterana and Oplonia microphylla. There were also: Malpighia linearis (very common), Guaiacum officinale, Eupatorium sinuatum and Gyminda latifolia. These species, which were among the most representative of the groundcover of the time, were associated with others already mentioned, specific to dry environments, some of which are calciphilous and common to insular areas with a calcareous substratum of the Guadeloupe archipelago (Marie-Galante, Grande-Terre, and the calcareous strip of Vieux-Fort (Basse-Terre)). Also indicated were associations of xerophytic open littoral environments with cacti (Melocactus intortus, Opuntia rubescens) and those which are mainly represented by crotons (Croton flavens. downwind region, Croton astroites. upwind region).

Vieux-Fort (Basse-Terre: H. Stehle, 1936)

As H. Stehle points out, the vegetation was located in a small area and constituted a relictual ecosystem of interest. Table 27 shows a group dominated by Pimenta racemosa and Cornutia pyramidata, with which other elective species were associated.

b) The area of the andesitic mornes of the lower region

In these territories, during the herborisation carried out by $\mathrm{H}$. Stehle (mid-1930s), the vegetation assumed a very marked xero-heliophilous appearance, presenting some similarities with that of the leeward littoral areas directly

${ }^{5}$ Formerly very abundant, became rare in this period (1936) following excessive exploitation. 
Table 26. Tropical seasonal evergreen vegetation.

\begin{tabular}{ccc}
\hline Plant species & Abundance & Stratigraphic level \\
\hline Canella winterana & ++++++ & $\mathrm{S} 1 / \mathrm{S} 2$ \\
Eupatorium sinuatum & + & $\mathrm{S} 3$ \\
Guaiacum officinale & + & $\mathrm{S} 1 / \mathrm{S} 2$ \\
Gyminda latifolia & + & $\mathrm{S} 1 / \mathrm{S} 2$ \\
Malpighia linearis & ++ & $\mathrm{S} 3$ \\
Oplonia microphylla & +++++ & $\mathrm{S} 3$ \\
\hline
\end{tabular}

(++++++): very high Abundance/(++++): high Abundance/(+++): medium Abundance/(++): low Abundance/(+): very low Abundance. S1: Upper stratum/S2: Medium stratum/S3: Lower stratum.

Table 27. Tropical seasonal evergreen vegetation.

\begin{tabular}{|c|c|c|c|c|c|}
\hline Plant species & Abundance & $\begin{array}{c}\text { Stratigraphic } \\
\text { level }\end{array}$ & Plant species & Abundance & $\begin{array}{c}\text { Stratigraphic } \\
\text { level }\end{array}$ \\
\hline $\begin{array}{c}\text { Pimenta } \\
\text { racemosa }\end{array}$ & ++++++ & $\mathrm{S} 1 / \mathrm{S} 3$ & Picramnia pentandra & + & S3 \\
\hline $\begin{array}{l}\text { Cornutia py- } \\
\text { ramidata }\end{array}$ & +++++ & S2/S3 & $\begin{array}{l}\text { Krugiodendron } \\
\text { ferreum }\end{array}$ & + & $\mathrm{S} 2 / \mathrm{S} 3$ \\
\hline $\begin{array}{l}\text { Malpighia } \\
\text { glabra }\end{array}$ & ++ & S3 & $\begin{array}{c}\text { Foresteria } \\
\text { rhamnifolia }\end{array}$ & + & $\mathrm{S} 2 / \mathrm{S} 3$ \\
\hline $\begin{array}{c}\text { Eugenia } \\
\text { ligustrina }\end{array}$ & ++ & S3 & $\begin{array}{c}\text { Erythroxylon } \\
\text { havanense }\end{array}$ & + & S3 \\
\hline $\begin{array}{l}\text { Zanthoxylum } \\
\text { punctatum }\end{array}$ & + & S3 & Brunfelsia americana & + & S3 \\
\hline
\end{tabular}

$(++++++)$ : very high Abundance/(++++): high Abundance/(+++): medium Abundance/(++): low Abundance/(+): very low Abundance. S1: Upper stratum/S2: Middle stratum/S3: Lower stratum.

exposed to the marine environment. Naturally, as for those which are currently developing under these eco-climatic conditions, the species outlined which are part of the various elementary groupings, present a fairly wide range of adaptive strategies, which relate to physiology (secretion of aromatic juices and essential oils, caducity), anatomy and morphology. According to the author, at this period the bush physiognomic type was the most frequent. By way of example and to demonstrate the ecosystemic diversity of these anthropised territories with andesitic substratum, we will cite a few corteges or species which were predominant in 1936 and which thus reflected a plurality of environments.

In Table 28, the predominant species in terms of their demography, distribution and biomass are, in order of significance: Bursera simaruba, Protium attenuatum, Lonchocarpus violaceus, Amyris elemifera and Erythroxylon havanense. The populations of these species are accompanied by those of Coccoloba pubescens, Daphnopsis Americana, Eugenia confusa, Homalium racemosum, Lonchocarpus pantaphyllus and Ocotea coriacea. The whole forms a canopy with non-overlapping crowns, and is therefore open.

Guadeloupe (Basse-Terre: H. Stehle, 1936): 
Table 28. Tropical seasonal evergreen vegetation.

\begin{tabular}{|c|c|c|c|c|c|}
\hline Plant species & Abundance & $\begin{array}{c}\text { Stratigraphic } \\
\text { level }\end{array}$ & Plant species & Abundance & $\begin{array}{c}\text { Stratigraphic } \\
\text { level }\end{array}$ \\
\hline Amyris elemifera & +++++ & S3 & $\begin{array}{l}\text { Homalium } \\
\text { racemosum }\end{array}$ & ++ & $\mathrm{S} 1 / \mathrm{S} 2$ \\
\hline Bursera simaruba & ++++++ & $\mathrm{S} 1 / \mathrm{S} 2$ & Licania ternatensis & + & $\mathrm{S} 2 / \mathrm{S} 3$ \\
\hline Calliandra prupurea & + & S3 & $\begin{array}{l}\text { Lonchocarpus } \\
\text { pantaphyllus }\end{array}$ & ++ & $\mathrm{S} 2 / \mathrm{S} 3$ \\
\hline Cestrum $s p$ & + & S3 & $\begin{array}{c}\text { Lonchocarpus } \\
\text { violaceus }\end{array}$ & +++++ & $\mathrm{S} 2 / \mathrm{S} 3$ \\
\hline $\begin{array}{l}\text { Coccoloba } \\
\text { pubescens }\end{array}$ & +++ & $\mathrm{S} 2 / \mathrm{S} 3$ & $\begin{array}{l}\text { Morisonia } \\
\text { Americana }\end{array}$ & + & S3 \\
\hline Coccoloba swartzii & ++ & $\mathrm{S} 2 / \mathrm{S} 3$ & Ocotea coriacea & ++ & $\mathrm{S} 2 / \mathrm{S} 3$ \\
\hline Coccoloba venosa & + & S3 & Ocotea eggersiana & + & $\mathrm{S} 1 / \mathrm{S} 2$ \\
\hline $\begin{array}{l}\text { Conostegia } \\
\text { calyptrata }\end{array}$ & + & S3 & Ouratea guildingii & ++ & S3 \\
\hline Cordia alliodora & ++ & $\mathrm{S} 2 / \mathrm{S} 1$ & $\begin{array}{l}\text { Pilocarpus } \\
\text { racemosus }\end{array}$ & ++ & S3 \\
\hline Croton corylifolius & + & S3 & Pouteria multifolia & + & $\mathrm{S} 1 / \mathrm{S} 2$ \\
\hline $\begin{array}{l}\text { Daphnopsis } \\
\text { americana }\end{array}$ & +++ & S3 & $\begin{array}{c}\text { Protium } \\
\text { attenuatum }\end{array}$ & ++++++ & $\mathrm{S} 1 / \mathrm{S} 2$ \\
\hline $\begin{array}{c}\text { Erythroxylon } \\
\text { havanense }\end{array}$ & +++++ & S3 & Rochefortia spinosa & + & $\mathrm{S} 2 / \mathrm{S} 3$ \\
\hline Eugenia confusa & +++ & S3 & $\begin{array}{c}\text { Tetrazygia } \\
\text { angustifolia }\end{array}$ & + & S3 \\
\hline $\begin{array}{c}\text { Eugenia } \\
\text { lambertiana }\end{array}$ & + & S3 & Vernonia albicaulis & + & S3 \\
\hline $\begin{array}{c}\text { Eugenia } \\
\text { pseudopsidium }\end{array}$ & + & S3 & $\begin{array}{l}\text { Vernonia } \\
\text { arborescens }\end{array}$ & + & S3 \\
\hline
\end{tabular}

(++++++): very high Abundance/(++++): high Abundance/(+++): medium Abundance/(++): low Abundance/(+): very low Abundance. S1: Upper stratum/S2: Middle stratum/S3: Lower stratum.

Some examples of association

- Association of Protium attenuatum (+++++), Bursera simaruba (++++) and Amyris elemifera $(+++)$.

- Association of Lonchocarpus violaceus $(+++++)$ and Bursera simaruba $(+++)$.

- Association of Lonchocarpus pentaphyllus (?, ++++) and Bursera simaruba $(+++)$.

- Association of Coccoloba swartzii (+++++), Coccoloba venosa (++++), Eugenia lambertiana $(+++)$, Eugenia monticola $(+++)$ and Ardisia obovata $(++)$.

- Formation dominated by Ouratea guildinguii $(+++++)$.

- Formation dominated by Cordia alliodora $(++++)$, with secondarily Croton corylifolius $(+++)$. 
- Formation dominated by Calliandra purpurea (+++++), with secondarily Cordia alliodora $(++++)$, Eugenia monticola $(+++)$, Lonchocarpus violaceus $(++)$ and Lonchocarpus pentaphyllus $(++)$.

- Formation dominated by Homalium racemosum (+++++).

Table 29 indicates another example of a floristic grouping where Protium attenuatum, Bursera simaruba and Amyris elemifera are dominant. In other words, they are more distributed spatially and have a higher aboveground population biomass.

\section{Les Saintes (Terre-de-Bas: H. Stehle, 1936)}

These very small islands are characterised by a long drought and most of the plant formations are in the young sylvatic stage. The predominant cortege mentioned in Table 30 is composed of Rochefortia spinosa and Eugenia ligustrina.

6) Guadeloupe (J.P. Fiard and B. Rollet, 1988): Les Saintes (Terre-de-bas)

Morne Abymes (altitude $296 \mathrm{~m}$ ) and plateau (altitude $260 \mathrm{~m}$ ) between Morne Abymes and Morne Sec (altitude $188 \mathrm{~m}$ ).

Grouping of predominant species:

Hymenaea courbaril, Pimenta racemosa, Inga laurina, Lonchocarpus violaceus.

\section{Dominant floristic set, upper stratum $(17 \mathrm{~m})$ :}

Hymenaea courbaril, Pimenta racemosa, Inga laurina, Lonchocarpus violaceus, Pisonia fragrans, Zanthoxylum flavum, Cordia sulcata, Zanthoxylum caribaeum, Bursera simaruba, Tabebuia heterophylla.

Dominated floristic set of greatest abundance, middle stratum (10 - 15 $\mathrm{m})$ :

Ocotea coriacea, Zanthoxylum monophyllum.

Dominated floristic set of second greatest abundance, lower stratum (3 $10 \mathrm{~m})$ :

\section{Zanthoxylum punctatum, Eugenia ligustrina.}

The floristic composition mentioned in Table 31 is typical of a tropical seasonal evergreen formation in its lower horizon subtype and in its late secondary dynamic stage. With respect to current data on the sylvatic diversity of the Lesser Antilles islands, each of which has its own factorial identity, small volcanic islets like Les Saintes only allow the development of the lower horizon subtype of the tropical seasonal evergreen sylva. The observable successive stages result from anthropisation. The analysis of the composition of the species in terms of temperament and ecological dominance reveals a structural state which does not reflect the real dynamic state. Indeed the main plant framework of this station is produced by two electives of the final stage installing from the presylvatic phase: Hymenaea courbaril and Pimenta racemosa. Given the inventory data, it appears that the regenerative capacity is more significant for the regressive species ${ }^{6}$ (bush or pre-forest). In reality, this formation derives from the mature sylva, while being edified by species which probably belonged to the original climax. The

${ }^{6}$ Temperament of primary or secondary pioneers. 
Table 29. Formation dominated by three edifying species.

\begin{tabular}{|c|c|c|c|c|c|}
\hline Plant species & Abundance & $\begin{array}{c}\text { Stratigraphic } \\
\text { level }\end{array}$ & $\begin{array}{c}\text { Plant } \\
\text { species }\end{array}$ & Abundance & $\begin{array}{c}\text { Stratigraphic } \\
\text { level }\end{array}$ \\
\hline Amyris elemifera & +++++ & S3 & Eugenia monticola & ++ & S3 \\
\hline Bursera simaruba & +++++ & $\mathrm{S} 1 / \mathrm{S} 2$ & $\begin{array}{c}\text { Exostema } \\
\text { sanctae-luciae }\end{array}$ & + & $\mathrm{S} 2 / \mathrm{S} 3$ \\
\hline Cestrum $s p$ & + & S3 & $\begin{array}{c}\text { Faramea } \\
\text { occidentalis }\end{array}$ & + & S3 \\
\hline $\begin{array}{l}\text { Cinnamomum } \\
\text { elongatum }\end{array}$ & + & $\mathrm{S} 2 / \mathrm{S} 3$ & Gyminda latifolia & + & $\mathrm{S} 2 / \mathrm{S} 3$ \\
\hline $\begin{array}{l}\text { Coccoloba } \\
\text { pubescens }\end{array}$ & + & $\mathrm{S} 2 / \mathrm{S} 1$ & Ocotea coriacea & ++ & $\mathrm{S} 2 / \mathrm{S} 3$ \\
\hline Cordia alliodora & +++ & $\mathrm{S} 2 / \mathrm{S} 1$ & $\begin{array}{c}\text { Protium } \\
\text { attenuatum }\end{array}$ & ++++++ & $\mathrm{S} 2 / \mathrm{S} 1$ \\
\hline Croton corylifolius & + & S3 & Vernonia albicaulis & + & S3 \\
\hline Croton $s p$ & + & S3 & $\begin{array}{c}\text { Vernonia } \\
\text { arborescens }\end{array}$ & + & S3 \\
\hline $\begin{array}{l}\text { Daphnopsis } \\
\text { americana }\end{array}$ & + & $\mathrm{S} 2 / \mathrm{S} 3$ & & & \\
\hline
\end{tabular}

$(++++++)$ : very high Abundance/(++++): high Abundance/(+++): medium Abundance/(++): low Abundance/(+): very low Abundance. S1: Upper stratum/S2: Middle stratum/S3: Lower stratum.

Table 30. Typical of a tropical seasonal evergreen formation.

\begin{tabular}{|c|c|c|c|c|c|}
\hline Plant species & Abundance & $\begin{array}{c}\text { Stratigraphic } \\
\text { levels }\end{array}$ & $\begin{array}{l}\text { Plant } \\
\text { species }\end{array}$ & Abundance & $\begin{array}{c}\text { Stratigraphic } \\
\text { levels }\end{array}$ \\
\hline Acacia muricata & + & S3 & Plumieria alba & + & S3 \\
\hline $\begin{array}{c}\text { Argythamnia } \\
\text { polygama }\end{array}$ & + & S3 & Rauvolfia viridis & + & S3 \\
\hline $\begin{array}{c}\text { Capparis cyno } \\
\text { phallophora }\end{array}$ & + & S3 & Rochefortia spinosa & ++++++ & $\mathrm{S} 2 / \mathrm{S} 3$ \\
\hline Capparis flexuosa & + & S3 & $\begin{array}{c}\text { Tabebuia } \\
\text { heterophylla }\end{array}$ & + & $\mathrm{S} 2 / \mathrm{S} 3 / \mathrm{S} 1$ \\
\hline Eugenia ligustrina & ++++ & S3 & Wedelia calycina & + & S3 \\
\hline $\begin{array}{l}\text { Malpighia } \\
\text { emarginata }\end{array}$ & + & S3 & $\begin{array}{c}\text { Zanyhoxylum } \\
\text { martinicense }\end{array}$ & + & $\mathrm{S} 3 / \mathrm{S} 2$ \\
\hline $\begin{array}{l}\text { Morisonia } \\
\text { americana }\end{array}$ & + & $\mathrm{S} 2 / \mathrm{S} 3$ & & & \\
\hline
\end{tabular}

$(++++++)$ : very high Abundance/(++++): high Abundance/(+++): medium Abundance/(++): low Abundance/(+): very low Abundance. S1: Upper stratum/S2: Middle stratum/S3: Lower stratum.

ecological changes due to the development of Les Saintes significantly reduced the regeneration capacity of these highly specialised and climactic species. Some authors clumsily call this ecosystemic state of the tropical seasonal evergreen sylva of lower horizon xerophytic secondary forest entity, when it quite simply corresponds to a transitional dynamic stage specific to the emergence of more general xerophilous plants. 
Table 31. Typical of a tropical seasonal evergreen formation.

\begin{tabular}{|c|c|c|c|c|c|}
\hline Plant species & Abundance & $\begin{array}{c}\text { Stratigraphic } \\
\text { levels }\end{array}$ & Plant species & Abundance & $\begin{array}{l}\text { Stratigraphic } \\
\text { levels }\end{array}$ \\
\hline Acacia tamarinfolia & + & S3 & Eugenia monticola & + & S3 \\
\hline $\begin{array}{c}\text { Actinostemon } \\
\text { caribaeus }\end{array}$ & + & S3 & Exothea caribaea & + & $\mathrm{S} 3 / \mathrm{S} 3$ \\
\hline $\begin{array}{l}\text { Allophyllus } \\
\text { racemosus }\end{array}$ & + & $\mathrm{S} 2 / \mathrm{S} 3$ & Ficus citrifolia & + & S2 \\
\hline Amyris elemifera & + & S3 & Guettarda scabra & + & $\mathrm{S} 2 / \mathrm{S} 3$ \\
\hline $\begin{array}{c}\text { Anacardium } \\
\text { occidentale }\end{array}$ & + & S3 & $\begin{array}{l}\text { Haematoxylon } \\
\text { campechianum }\end{array}$ & + & $\mathrm{S} 2 / \mathrm{S} 3$ \\
\hline Annona muricata & + & S3 & $\begin{array}{l}\text { Hippomane manci- } \\
\text { nella }\end{array}$ & + & $\mathrm{S} 2 / \mathrm{S} 1$ \\
\hline Annona squamosa & + & S3 & Hymenaea courbaril & $l \quad++++++$ & $\mathrm{S} 1 / \mathrm{S} 2$ \\
\hline Bontia daphnoides & + & S3 & Inga laurina & ++++ & $\mathrm{S} 2 / \mathrm{S} 1$ \\
\hline Bourreria succulenta & + & $\mathrm{S} 2 / \mathrm{S} 3$ & Jaquinia armillaris & + & S3 \\
\hline Bromelia plumieri & + & S3 & $\begin{array}{l}\text { Krugiodendron } \\
\text { ferreum }\end{array}$ & + & $\mathrm{S} 2 / \mathrm{S} 3$ \\
\hline Bursera simaruba & ++ & $\mathrm{S} 1 / \mathrm{S} 2$ & $\begin{array}{c}\text { Lonchocarpus vi- } \\
\text { olaceus }\end{array}$ & ++++ & $\mathrm{S} 1 / \mathrm{S} 2$ \\
\hline Byrsonima spicata & + & $\mathrm{S} 1 / \mathrm{S} 2$ & Mangifera indica & + & S1 \\
\hline Calliandra purpurea & + & S3 & Maytenus laevigata & + & $\mathrm{S} 2 / \mathrm{S} 3$ \\
\hline Capparis baducca & + & S3 & $\begin{array}{c}\text { Melicoccus } \\
\text { bijugatus }\end{array}$ & + & S3 \\
\hline Capparis flexuosa & + & S3 & $\begin{array}{l}\text { Morisonia } \\
\text { americana }\end{array}$ & + & S3 \\
\hline Capparis indica & + & S3 & Ocotea coriacea & + & $\mathrm{S} 2 / \mathrm{S} 3$ \\
\hline Casearia decandra & + & $\mathrm{S} 2 / \mathrm{S} 3$ & Pimenta racemosa & ++++++ & $\mathrm{S} 1 / \mathrm{S} 2$ \\
\hline Cedrela odorata & + & $\mathrm{S} 1 / \mathrm{S} 2$ & $\begin{array}{c}\text { Piscidia } \\
\text { carthagenensis }\end{array}$ & + & $\mathrm{S} 2 / \mathrm{S} 3$ \\
\hline Ceiba pentandra & + & S1 & Pisonia aculeata & + & S3 \\
\hline Celtis iguanaea & + & S3 & Pisonia fragrans & ++ & $\mathrm{S} 2 / \mathrm{S} 3$ \\
\hline Chiococca alba & + & S3 & Plumieria alba & + & S3 \\
\hline $\begin{array}{c}\text { Citharexylum } \\
\text { spinosum }\end{array}$ & + & $\mathrm{S} 3 / \mathrm{S} 2$ & $\begin{array}{l}\text { Psychotria } \\
\text { microdon }\end{array}$ & + & S3 \\
\hline Coccoloba pubescens & + & $\mathrm{S} 2 / \mathrm{S} 3 / \mathrm{S} 1$ & Randia aculeata & + & S3 \\
\hline Coccoloba venosa & + & S3 & $\begin{array}{c}\text { Rochefortia } \\
\text { acanthophora }\end{array}$ & + & $\mathrm{S} 2 / \mathrm{S} 3$ \\
\hline $\begin{array}{l}\text { Colubrina } \\
\text { arborescens }\end{array}$ & + & $\mathrm{S} 2 / \mathrm{S} 3$ & Schoepfia schreberi & + & S3 \\
\hline Cordia collococca & + & $\mathrm{S} 2 / \mathrm{S} 3$ & Spondias mombin & + & $\mathrm{S} 1 / \mathrm{S} 2$ \\
\hline Cordia martinicensis & + & S3 & Swietenia mahagoni & + & $\mathrm{S} 1 / \mathrm{S} 2$ \\
\hline Cordia sulcata & ++ & $\mathrm{S} 2 / \mathrm{S} 1$ & $\begin{array}{c}\text { Tabebuia } \\
\text { heterophylla }\end{array}$ & + & $\mathrm{S} 1 / \mathrm{S} 2$ \\
\hline
\end{tabular}




\begin{tabular}{|c|c|c|c|c|c|}
\hline \multicolumn{6}{|l|}{ Continued } \\
\hline Croton corylifolius & + & S3 & $\begin{array}{c}\text { Tabernaemontana } \\
\text { citrifolia }\end{array}$ & + & S3 \\
\hline Croton flavens & + & S3 & Triphasia trifolia & + & S3 \\
\hline $\begin{array}{l}\text { Erythroxylon } \\
\text { havanense }\end{array}$ & + & S3 & $\begin{array}{c}\text { Vangueria } \\
\text { madagascariensis }\end{array}$ & + & S3 \\
\hline $\begin{array}{c}\text { Eugenia } \\
\text { chrysobalanoides }\end{array}$ & + & S3 & $\begin{array}{c}\text { Zanthoxylum } \\
\text { caribaeum }\end{array}$ & ++ & S2/S3 \\
\hline Eugenia cordata & + & S3 & $\begin{array}{c}\text { Zanthoxylum } \\
\text { lavum }\end{array}$ & ++ & $\mathrm{S} 2 / \mathrm{S} 1$ \\
\hline Eugenia hodgei & + & S3 & $\begin{array}{l}\text { Zanthoxylum } \\
\text { monophyllum }\end{array}$ & + & S3 \\
\hline \multirow[t]{2}{*}{ Eugenia ligustrina } & + & S3 & $\begin{array}{l}\text { Zanthoxylum } \\
\text { punctatum }\end{array}$ & + & S3 \\
\hline & & & Ziziphus mauritiana & + & S3 \\
\hline
\end{tabular}

$(++++++)$ : very high Abundance/(++++): high Abundance/(+++): medium Abundance/(++): low Abundance/(+): very low Abundance. S1: Upper stratum/S2: Middle stratum/S3: Lower stratum.

7) P. Joseph and J.P. Fiard (1990): Les Saintes, Terre-de-Haut (Le Chameau, southern slope, survey at $600 \mathrm{~m}^{2}$ )

Grouping of structuring and predominant species: Busera simaruba, Lonchocarpus violaceus, Hymenaea courbaril and Sideroxylon foetidissimum.

A relictual forest unit, which once covered the whole of the Les Saintes archipelago. Although currently in a regressive state, it contains edifying species from the climactic final stage such as Sideroxylon foetidissimum (False Mastic of two metres in diameter). This sylva is highly significant, because it allows us to specify the potential sylvatic type of these regions subjected to the dry bioclimate. Admittedly, nowadays, the vegetation of Terre-de-Haut is completely ruined and the most frequent phytocenoses are of the shrubland or bush type. The relictual sylvan islets testify to the ecosystemic state of the primitive Les Saintes groundcover which should have corresponded to the tropical seasonal evergreen sylva in its lower horizon sub-type. The eco-units of the present are essentially engaged in dynamic processes belonging to the extra-sylvatic successional cycles. The resulting vegetation is low and open. In the exposed areas of the littoral, it presents a pronounced xeromorphic aspect which constitutes the extremity, almost irreversible, of the degradation of the original plant systems (Table 32).

\section{8) P Joseph (1997, Martinique)}

The following data are the result of surveys carried out in forest formations of the lower stage in Martinique. The diversity of the plant corteges corresponds to a phenological and physiognomic diversity. The floristic mosaic is dense. Each ecological unit is a regressive form and therefore transitional with respect to those which existed in the pre-Columbian period.

The forest in the lowlands of Martinique today occupies a small area and is highly heterogeneous. The numerous phytocenoses are mainly composed of regressive species as well as, depending on the stational eco-climatic conditions, 
Table 32. Relictual forest unit.

\begin{tabular}{|c|c|c|c|c|c|}
\hline Plant species & Abundance & $\begin{array}{c}\text { Stratigraphic } \\
\text { levels }\end{array}$ & Plant species & Abundance & $\begin{array}{c}\text { Stratigraphic } \\
\text { levels }\end{array}$ \\
\hline $\begin{array}{l}\text { Allophyllus } \\
\text { racemosus }\end{array}$ & + & S3/S2 & Hymenaea courbaril & +++ & $\mathrm{S} 1 / \mathrm{S} 2$ \\
\hline Amyris elemifera & + & S3 & Lonchocarpus violaceus & +++++ & $\mathrm{S} 2 / \mathrm{S} 1$ \\
\hline $\begin{array}{l}\text { Bourreria } \\
\text { succulenta }\end{array}$ & + & S3 & Ocotea coroacea & + & $\mathrm{S} 2 / \mathrm{S} 3 / \mathrm{S} 1$ \\
\hline Bursera simaruba & ++++++ & $\mathrm{S} 1 / \mathrm{S} 2$ & Pimenta racemosa & ++ & $\mathrm{S} 1 / \mathrm{S} 2$ \\
\hline $\begin{array}{l}\text { Citharexylum } \\
\text { spinosum }\end{array}$ & + & $\mathrm{S} 2 / \mathrm{S} 3$ & Pisonia fragrans & ++ & $\mathrm{S} 2 / \mathrm{S} 3 / \mathrm{S} 1$ \\
\hline $\begin{array}{c}\text { Erythroxylon } \\
\text { havanense }\end{array}$ & + & S3 & Randia aculeata & + & S3 \\
\hline Eugenia ligustrina & + & S3 & $\begin{array}{l}\text { Sideroxylon foetidissi- } \\
\text { mum }\end{array}$ & +++ & $\mathrm{S} 2 / \mathrm{S} 3 / \mathrm{S} 1$ \\
\hline $\begin{array}{l}\text { Exostema } \\
\text { caribaeum }\end{array}$ & + & $\mathrm{S} 2 / \mathrm{S} 3$ & $\begin{array}{c}\text { Zanthoxylum } \\
\text { microcarpum (?) }\end{array}$ & + & S3 \\
\hline
\end{tabular}

$(++++++)$ : very high Abundance/(++++): high Abundance/(+++): medium Abundance/(++): low Abundance/(+): very low Abundance. S1: Upper stratum/S2: Middle stratum/S3: Lower stratum.

late and final secondary species such as Maytenus laevigata, Sideroxylon foetidissimum, Manilkara bidentata, Guarea glabra, Licaria sericea, Buchenavia tetraphylla, Pouteria semecarpifolia (Table 33).

\subsection{Global Analysis of Table Data Using a CFA and an Ascending Hierarchical Classification (AHC)}

The previous tables show different population data corresponding to minimum areas of floristic inventories. In other words, even if these are not equivalent, they provide information on the totality of each groundcover taken as a reference. The Correspondence Factor Analysis is based on a table of abundance (cross table) composed of 237 rows (species) and 34 columns (tables or inventories) [42] [43]. There is a link between the rows and the columns since the $\mathrm{p}$-value (0.0001) calculated is lower than the alpha significance level (0.05). The F1 and F2 axes of Figure 2 show the TAB 26 and TAB 30 inventories which typically correspond with regard to the plant succession at the bush and sylvatic stage (Table 34) and which by their considerable floristic specificity focuses the other inventories (Table 34) practically at the start point of these factorial axes. This group of stations at the axes' start point presents differences.

By subtracting the TAB 26 and TAB 30 inventories from the data table, the new Correspondence Factor Analysis (CFA) highlights a group of presylvatic phytocenoses belonging to the extra-sylvatic dynamic phases and another affine of the sylvatic stages linked to the intra-sylvatic phases. On the F1 axis, the TAB 10 inventory distinction is to be related to its floristic specificities (Figure 3(a)). 
Table 33. Structured secondary sylvatic.

\begin{tabular}{|c|c|c|c|c|c|}
\hline $\begin{array}{l}\text { Plant } \\
\text { species }\end{array}$ & Abundance & $\begin{array}{c}\text { Stratigraphic } \\
\text { levels }\end{array}$ & $\begin{array}{l}\text { Plant } \\
\text { species }\end{array}$ & Abundance & $\begin{array}{c}\text { Stratigraphic } \\
\text { levels }\end{array}$ \\
\hline Pimenta racemosa & ++++++ & $\mathrm{S} 1 / \mathrm{S} 2$ & Ficus nymphaeifolia & + & S1 \\
\hline Ocotea coriacea & ++++++ & $\mathrm{S} 3 / \mathrm{S} 2$ & Eugenia axillaris & + & S3 \\
\hline Maytenus laevigata & ++++++ & $\mathrm{S} 2 / \mathrm{S} 3$ & $\begin{array}{c}\text { Buchenavia } \\
\text { tetraphylla }\end{array}$ & + & S3 \\
\hline $\begin{array}{c}\text { Sideroxylon } \\
\text { foetidissimum }\end{array}$ & +++++ & $\mathrm{S} 2 / \mathrm{S} 1$ & $\begin{array}{c}\text { Tabebuia } \\
\text { heterophylla }\end{array}$ & + & $\mathrm{S} 2 / \mathrm{S} 1$ \\
\hline Myrcia fallax & +++ & S2 & $\begin{array}{l}\text { Daphnopsis } \\
\text { americana }\end{array}$ & + & $\mathrm{S} 3 / \mathrm{S} 2$ \\
\hline Inga laurina & +++ & $\mathrm{S} 2 / \mathrm{S} 1$ & Exothea paniculata & + & $\mathrm{S} 2 / \mathrm{S} 3$ \\
\hline Pisonia fragrans & +++ & $\mathrm{S} 2 / \mathrm{S} 1$ & $\begin{array}{c}\text { Pouteria } \\
\text { semecarpifolia }\end{array}$ & + & $\mathrm{S} 2 / \mathrm{S} 1$ \\
\hline Bursera simaruba & ++ & $\mathrm{S} 2 / \mathrm{S} 1$ & $\begin{array}{c}\text { Eugenia } \\
\text { oerstedeana }\end{array}$ & + & S3 \\
\hline $\begin{array}{l}\text { Chrysophyllum } \\
\text { argenteum }\end{array}$ & ++ & $\mathrm{S} 2 / \mathrm{S} 3$ & Simaruba amara & + & $\mathrm{S} 2 / \mathrm{S} 3$ \\
\hline Manilkara bidentata & ++ & $\mathrm{S} 1 / \mathrm{S} 2$ & Capparis indica & + & $\mathrm{S} 2 / \mathrm{S} 3$ \\
\hline $\begin{array}{c}\text { Eugenia } \\
\text { pseudopsidium }\end{array}$ & ++ & S2 & Ocotea cernua & + & $\mathrm{S} 2 / \mathrm{S} 3$ \\
\hline $\begin{array}{l}\text { Pilocarpus } \\
\text { racemosus }\end{array}$ & ++ & S3 & $\begin{array}{l}\text { Haematoxylon } \\
\text { campechianum }\end{array}$ & + & $\mathrm{S} 2 / \mathrm{S} 3$ \\
\hline Coccoloba swartzii & + & $\mathrm{S} 2 / \mathrm{S} 3$ & Ilex nitida & + & \\
\hline Mangifera indica & + & S1 & Antirhea coriacea & $\mathrm{n}$ & $\mathrm{S} 3 / \mathrm{S} 2$ \\
\hline Ocotea eggersiana & + & S1 & Ficus citrifolia & + & $\mathrm{S} 2 / \mathrm{S} 1$ \\
\hline $\begin{array}{c}\text { Chionanthus } \\
\text { compacta }\end{array}$ & + & $\mathrm{S} 2 / \mathrm{S} 3$ & $\begin{array}{l}\text { Brosimum } \\
\text { alicastrum }\end{array}$ & + & $\mathrm{S} 2 / \mathrm{S} 1$ \\
\hline Guarea glabra & + & $\mathrm{S} 2 / \mathrm{S} 3$ & Guazuma ulmifolia & + & $\mathrm{S} 2 / \mathrm{S} 3$ \\
\hline Ocotea patens & + & $\mathrm{S} 2 / \mathrm{S} 3$ & Casearia decandra & + & $\mathrm{S} 3 / \mathrm{S} 2$ \\
\hline Faramea occidentalis & + & S3 & $\begin{array}{l}\text { Sideroxylon } \\
\text { obovatum }\end{array}$ & + & $\mathrm{S} 2 / \mathrm{S} 3$ \\
\hline Plinia pinnata & + & S3 & $\begin{array}{c}\text { Exostema } \\
\text { sanctae-luciae }\end{array}$ & + & $\mathrm{S} 2 / \mathrm{S} 3$ \\
\hline Spondias monbin & + & S1 & Sterculia caribaea & + & $\mathrm{S} 2 / \mathrm{S} 1$ \\
\hline $\begin{array}{l}\text { Cassipourea } \\
\text { guianensis }\end{array}$ & + & $\mathrm{S} 2 / \mathrm{S} 3$ & Andira inermis & + & $\mathrm{S} 2 / \mathrm{S} 3$ \\
\hline Eugenia monticola & + & S3 & $\begin{array}{l}\text { Zanthoxylum } \\
\text { flavum }\end{array}$ & + & $\mathrm{S} 1 / \mathrm{S} 2$ \\
\hline Guarea macrophylla & + & $\mathrm{S} 2 / \mathrm{S} 3$ & $\begin{array}{c}\text { Hymenaea } \\
\text { courbaril }\end{array}$ & + & $\mathrm{S} 2 / \mathrm{S} 1$ \\
\hline Eugenia ligustrina & + & S3 & $\begin{array}{l}\text { Coccoloba } \\
\text { pubescens }\end{array}$ & + & $\mathrm{S} 2 / \mathrm{S} 1$ \\
\hline Eugenia confusa & + & S3 & $\begin{array}{c}\text { Eugenia } t \\
\text { apacumensis }\end{array}$ & + & $\mathrm{S} 3 / \mathrm{S} 2$ \\
\hline Quararibea turbinata & + & $\mathrm{S} 3 / \mathrm{S} 2$ & $\begin{array}{c}\text { Lonchocarpus } \\
\text { vioalaceus }\end{array}$ & + & $\mathrm{S} 2 / \mathrm{S} 3$ \\
\hline
\end{tabular}




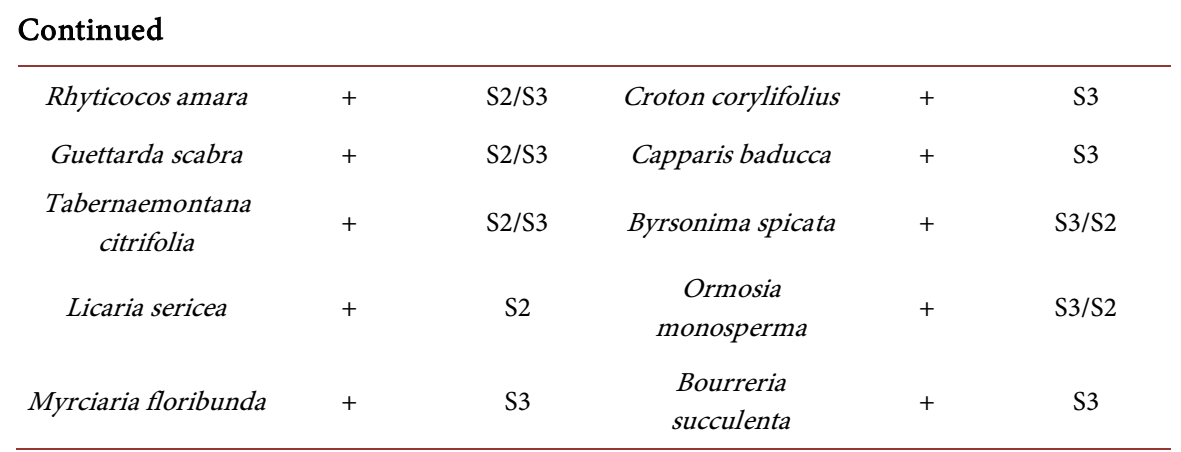

$(++++++)$ : very high Abundance/(++++): high Abundance/(+++): medium Abundance/(++): low Abundance/(+): very low Abundance. S1: Upper stratum/S2: Middle stratum/S3: Lower stratum.

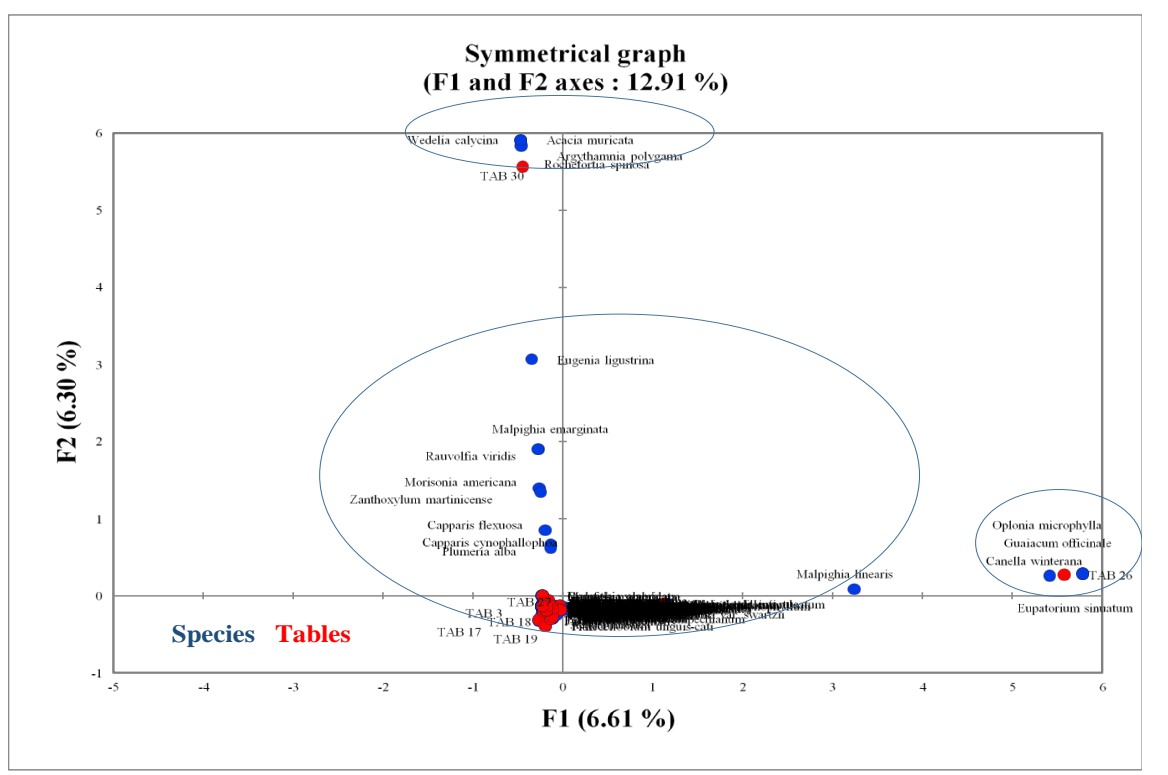

Figure 2. Comparison of the different surveys with regard to the population specificity of the species.

Whether these are pre-sylvatic or sylvatic groupings, the distances in the plane formed by the axes F1 and F2 of Figure 3(a) \& Figure 3(b) between the points symbolising the surveys and the determining species associated with them indicate both qualitative (species) and quantitative differences (population abundances) and a diversity of ecological profiles related to the biotic and physical factors.

An Ascending Hierarchical Classification (AHC) carried out using a table of abundance of 237 species from 34 inventories (TAB) and based on the general similarity according to the "Complete Linkage" aggregation method shows a multitude of degrees of similarity (Figure 4) [44] [45]. Except for the TAB 33 surveys, the floristic inventories have a similarity of between $60 \%$ and $65 \%$ (Figure 4 ). These differences in similarity ranging from $62 \%$ to $92 \%$ on average result from the combination of several parameters involved in the installation of species such as the geomorphological specificities of the different Lesser Antilles considered in this study which engender a plurality of topographical facies, 
Table 34. Bioclimates, ecosystemic potentialities and evolutionary stages.

\begin{tabular}{|c|c|c|}
\hline $\begin{array}{c}\text { Tables } \\
\text { (Stations) }\end{array}$ & Evolutionary stages & Bioclimates/Ecosystemic potentialities \\
\hline 1 & $\begin{array}{l}\text { Presylvatic or } \\
\text { young sylvatic }\end{array}$ & Dry subhumid/Tropical seasonal evergreen of lower horizon \\
\hline 2 & $\begin{array}{l}\text { Presylvatic or } \\
\text { young sylvatic }\end{array}$ & Dry subhumid/Tropical seasonal evergreen of lower horizon \\
\hline 3 & Presylvatic & $\begin{array}{l}\text { Dry subhumid (hyper-xeric facies)/Tropical seasonal ever- } \\
\text { green of lower horizon }\end{array}$ \\
\hline 4 & Presylvatic & Dry subhumid/Tropical seasonal evergreen of lower horizon \\
\hline 5 & Presylvatic & Dry subhumid/Tropical seasonal evergreen of lower horizon \\
\hline 6 & Secondary sylvatic & Humid subhumid/Tropical seasonal evergreen \\
\hline 7 & Presylvatic & Dry subhumid/Tropical seasonal evergreen of lower horizon \\
\hline 8 & Subclimactic sylvatic & $\begin{array}{l}\text { Humid/Seasonal evergreen forest ecotone/sub-montane } \\
\text { ombrophilous }\end{array}$ \\
\hline 9 & Presylvatic & $\begin{array}{l}\text { Dry subhumid (hyper-xeric facies)/Tropical seasonal ever- } \\
\text { green of lower horizon }\end{array}$ \\
\hline 10 & Presylvatic & $\begin{array}{l}\text { Dry subhumid (hyper-xeric facies)/Tropical seasonal ever- } \\
\text { green of lower horizon }\end{array}$ \\
\hline 11 & Presylvatic & $\begin{array}{l}\text { Dry subhumid (hyper-xeric facies)/Tropical seasonal ever- } \\
\text { green of lower horizon }\end{array}$ \\
\hline 12 & Presylvatic & $\begin{array}{l}\text { Dry subhumid (hyper-xeric facies)/Tropical seasonal ever- } \\
\text { green of lower horizon }\end{array}$ \\
\hline 13 & Presylvatic & Dry subhumid/Tropical seasonal evergreen of lower horizon \\
\hline 14 & Young sylvatic & Dry subhumid/Tropical seasonal evergreen of lower horizon \\
\hline 15 & $\begin{array}{l}\text { Presylvatic or } \\
\text { young sylvatic }\end{array}$ & Dry subhumid/Tropical seasonal evergreen of lower horizon \\
\hline 16 & $\begin{array}{c}\text { Structured } \\
\text { secondary sylvatic }\end{array}$ & Dry subhumid/Tropical seasonal evergreen of lower horizon \\
\hline 17 & Presylvatic & Dry subhumid/Tropical seasonal evergreen of lower horizon \\
\hline 18 & Secondary sylvatic & Dry subhumid/Tropical seasonal evergreen of lower horizon \\
\hline 19 & Presylvatic & Dry subhumid/Tropical seasonal evergreen of lower horizon \\
\hline 20 & Secondary sylvatic & Dry subhumid/Tropical seasonal evergreen of lower horizon \\
\hline 21 & $\begin{array}{c}\text { Structured } \\
\text { secondary sylvatic }\end{array}$ & Dry subhumid/Tropical seasonal evergreen of lower horizon \\
\hline 22 & $\begin{array}{c}\text { Structured } \\
\text { secondary sylvatic }\end{array}$ & Dry subhumid/Tropical seasonal evergreen of lower horizon \\
\hline 23 & Secondary sylvatic & Dry subhumid/Tropical seasonal evergreen of lower horizon \\
\hline 24 & Young sylvatic & Dry subhumid/Tropical seasonal evergreen of lower horizon \\
\hline 25 & Presylvatic & Dry subhumid/Tropical seasonal evergreen of lower horizon \\
\hline 26 & Bush & Dry subhumid/Tropical seasonal evergreen of lower horizon \\
\hline 27 & Secondary sylvatic & Dry subhumid/Tropical seasonal evergreen of lower horizon \\
\hline 28 & Secondary sylvatic & Dry subhumid/Tropical seasonal evergreen of lower horizon \\
\hline
\end{tabular}




\section{Continued}

\begin{tabular}{ccl}
\hline 29 & Secondary sylvatic & Dry subhumid/Tropical seasonal evergreen of lower horizon \\
30 & Young sylvatic & Dry subhumid/Tropical seasonal evergreen of lower horizon \\
31 & $\begin{array}{c}\text { Structured secondary } \\
\text { sylvatic }\end{array}$ & Dry subhumid/Tropical seasonal evergreen of lower horizon \\
32 & Secondary sylvatic & Dry subhumid/Tropical seasonal evergreen of lower horizon \\
33 & $\begin{array}{c}\text { Structured secondary } \\
\text { sylvatic }\end{array}$ & $\begin{array}{l}\text { Humid/Seasonal evergreen forest ecotone/sub-montane } \\
\text { ombrophilous }\end{array}$ \\
\hline
\end{tabular}

Symmetric column graph

(F1 and F2 axes: 13.34\%)

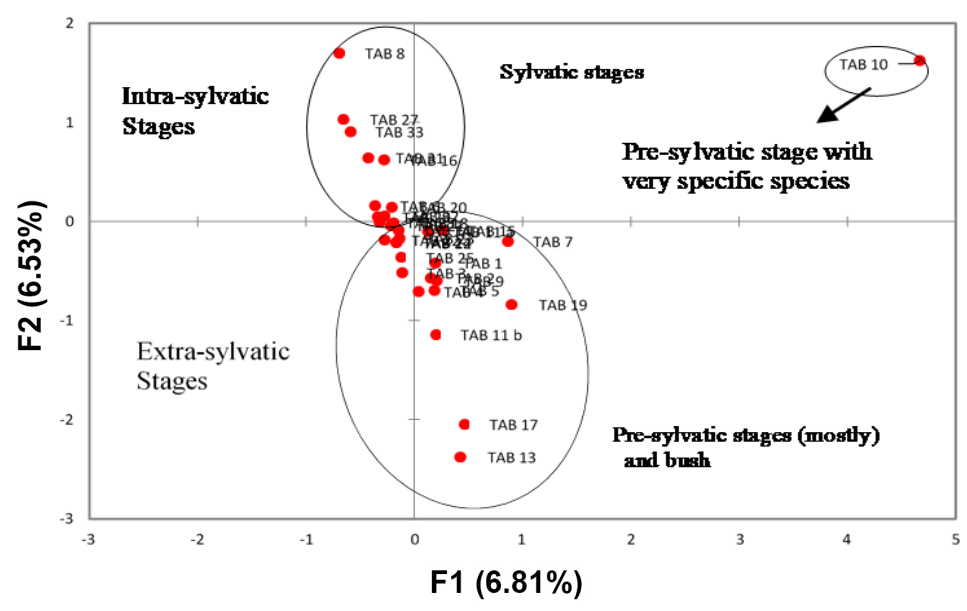

- Tables

(a)

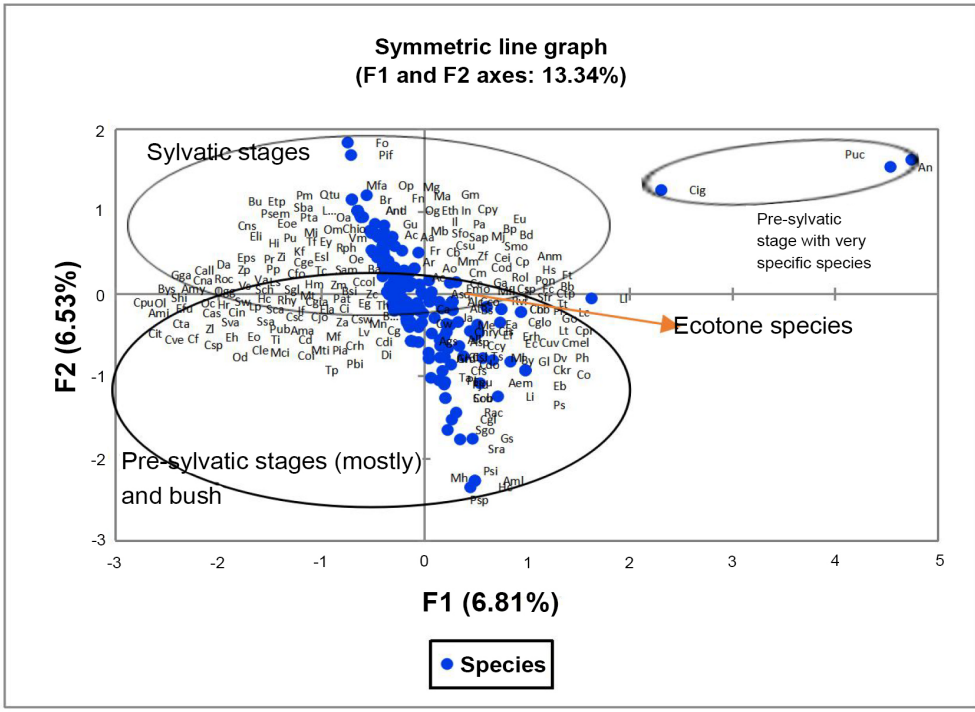

(b)

Figure 3. (a) Relation of surveys with regard to the population structure of the species; (b) Relation of the species of the different surveys with regard to their population structure [see Annex 1 (List of species abbreviations)]. 


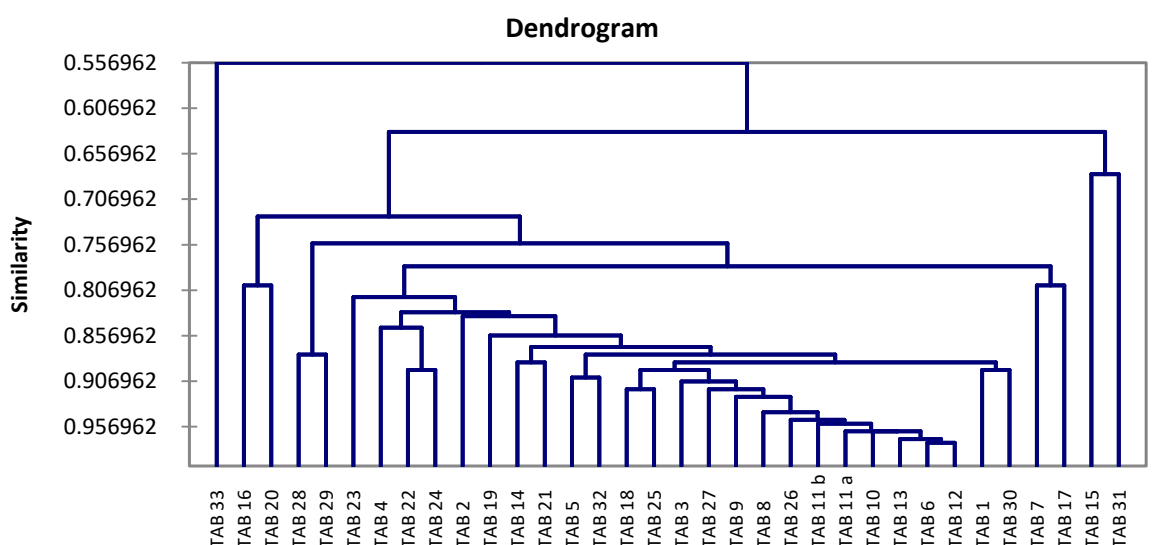

Figure 4. Degrees of similarity related to the types of species and their population structure.

bioclimatic affinities, edaphic characteristics, stages of ecosystemic evolution and spatiotemporal modalities of human activities (Figure 4). The topographic facets lead to mesoclimates subdivided into microclimates.

\section{Discussion and Conclusion}

The phytocenoses mentioned above, mainly through tables, are some examples of great diversity. The predominant species, both from a bio-demographic and ecological point of view, show the evolutionary stage of these phytocenoses. The above data highlight the considerable plasticity of the vegetation of the Lesser Antilles resulting from numerous floristic combinations. Indeed the contrasting geomorphology which leads to a multiplicity of topographical facets, the meso-climates ranging from dry to hyper-humid, the highly diverse edaphic systems resulting from these and the phases of the plant dynamics as well as the wind, in particular its impact at the littoral and high peaks, are the main factors behind the multiple habitats and therefore biodiversity, both specific and community [5] [46] [47] [48] [49]. Added to this are the phenomena of vegetation inversions which at the level of the peaks and ridges exposed to the wind as well as in valleys or dales increase the density of biotopes and therefore the potential for the colonisation of singular corteges of species. On the peaks and ridges of the humid bioclimate (middle and upper stages) exposed to wind and often shallow soils, evapotranspiration dries the environment and allows for the installation of affine species which thrive in conditions of the dry bioclimate (lower plant floor). Conversely, in the dales and valleys subject to the dry bioclimate (lower stage), the confinement which reduces the duration of sunshine and the colluviation due to the terrigenous erosion of the opposite slopes which considerably increases the depth of the soil leads to the installation of plant groups typical of moderately humid or even humid zones whose ecosystemic potentialities are characteristically the tropical seasonal evergreen sylva and the tropical submontane ombrophilous sylva. From one island to the next, anthropisation, which varies in terms of intensity and frequency, has led to and is still leading to 
a greater diversification of environments hosting a plurality of phytocenoses of variable areal significance. In general, it can be said that mesological factors with complex structures and anthropisation which varies as regards time and space are the drivers of the great diversity of biotopes and therefore phytocenoses. More than in pre-Columbian times, the anthropised Lesser Antilles of today can be considered as highly differentiated floristic assemblages. Each insular groundcover can give rise to a dense mosaic of phytocenoses of varying spatial significance, age, structure, architecture and floristic composition. A synchronic analysis makes it possible to reconstruct the evolutionary stages and to specify for the hyperhumid, humid, humid subhumid and dry subhumid bioclimates the ecosystemic potentialities and the various dominant plant combinations. Ultimately, in view of the above, the phytocenotic canvas typical of each Lesser Antilles, each unit of which is a biosystemic response, represents a veritable laboratory for the study of plant succession, a complex non-linear phenomenon of which the spatiotemporal characteristics remain to be deciphered.

\section{Acknowledgements}

Our thanks go first to the members of the Antilles "BIORECA" group from the "UMR ESPACE DEV" laboratory and secondly to the CTM (Local Authority of Martinique) and the University of the Antilles which annually fund our research programmes.

\section{Conflicts of Interest}

The authors declare no conflicts of interest regarding the publication of this paper.

\section{References}

[1] Veblen, K.E. and Young, T.P. (2010) Contrasting Effects of Cattle and Wildlife on the Vegetation Development of a Savanna Landscape Mosaic. Journal of Ecology, 98, 993-1001. https://doi.org/10.1111/j.1365-2745.2010.01705.x

[2] del Arco Aguilar, M.J., González-González, R., Garzón-Machado, V. and Pizarro-Hernández, B. (2010) Actual and Potential Natural Vegetation on the Canary Islands and Its Conservation Status. Biodiversity and Conservation, 19, 3089-3140. https://doi.org/10.1007/s10531-010-9881-2

[3] Wu, G.L., Wang, D., Liu, Y., Hao, H.M., Fang, N.F. and Shi, Z.H. (2016) Mosaic-Pattern Vegetation Formation and Dynamics Driven by the Water-Wind Crisscross Erosion. Journal of Hydrology, 538, 355-362.

https://doi.org/10.1016/j.jhydrol.2016.04.030

[4] Burga, C.A., Krüsi, B., Egli, M., Wernli, M., Elsener, S., Ziefle, M., Mavris, C., et al. (2010) Plant Succession and Soil Development on the Foreland of the Morteratsch Glacier (Pontresina, Switzerland): Straight Forward or Chaotic? Flora-Morphology, Distribution, Functional Ecology of Plants, 205, 561-576. https://doi.org/10.1016/j.flora.2009.10.001

[5] Bu, W., Zang, R. and Ding, Y. (2014) Functional Diversity Increases with Species Diversity along Successional Gradient in a Secondary Tropical Lowland Rainforest. 
Tropical Ecology, 55, 393-401. https://doi.org/10.1016/j.actao.2013.10.002

[6] Klanderud, K., Mbolatiana, H.Z.H., Vololomboahangy, M.N., Radimbison, M.A., Roger, E., Totland, Ø. and Rajeriarison, C. (2010) Recovery of Plant Species Richness and Composition after Slash-and-Burn Agriculture in a Tropical Rainforest in Madagascar. Biodiversity and Conservation, 19, 187.

https://doi.org/10.1007/s10531-009-9714-3

[7] Lohbeck, M., Poorter, L., Martínez-Ramos, M., Rodriguez-Velázquez, J., van Breugel, M. and Bongers, F. (2014) Changing Drivers of Species Dominance during Tropical Forest Succession. Functional Ecology, 28, 1052-1058. https://doi.org/10.1111/1365-2435.12240

[8] Arroyo-Rodríguez, V., Melo, F.P., Martínez-Ramos, M., Bongers, F., Chazdon, R.L., Meave, J.A., Tabarelli, M., et al. (2017) Multiple Successional Pathways in Human-Modified Tropical Landscapes: New Insights from Forest Succession, Forest Fragmentation and Landscape Ecology Research. Biological Reviews, 92, 326-340. https://doi.org/10.1111/brv.12231

[9] Salama, F.M., El Naggar, S.M. and Baayo, K.A. (2005) Vegetation Structure and Environmental Gradients in the Sallum Area, Egypt. Ecologia Mediterranea, 31, 15-32.

[10] Noumi, E. (2013) Floristic Inventory of Woody Species in the Manengouba Mountain Forest, Cameroon. Journal of Biology and Life Science, 4, 282-309. https://doi.org/10.5296/jbls.v4i2.4014

[11] Noumi, E. (2015) Floristic Structure and Diversity of a Tropical Sub-Montane Evergreen Forest, in the Mbam Minkom Massif (Western Yaoundé). Journal of Biology and Life Sciences, 6, 149. https://doi.org/10.5296/jbls.v6i1.7028

[12] Baruch, Z. (1984) Ordination and Classification of Vegetation along an Altitudinal Gradient in the Venezuelan páramos. Vegetatio, 55, 115-126.

[13] Trifanov, C., Romanescu, G., Tudor, M., Grigoras, I., Doroftei, M., Covaliov, S. and Mierla, M. (2018) Anthropisation Degree of Coastal Vegetation Areas in Danube Delta Biosphere Reserve. Journal of Environmental Protection and Ecology, 19, 539-546.

[14] Derroire, G., Balvanera, P., Castellanos-Castro, C., Decocq, G., Kennard, D.K., Lebrija-Trejos, E., Tigabu, M., et al. (2016) Resilience of Tropical Dry Forests: A Meta-Analysis of Changes in Species Diversity and Composition during Secondary Succession. Oikos, 125, 1386-1397. https://doi.org/10.1111/oik.03229

[15] Jamoneau, A., Sonnier, G., Chabrerie, O., Closset-Kopp, D., Saguez, R., Gallet-Moron, E. and Decocq, G. (2011) Drivers of Plant Species Assemblages in Forest Patches among Contrasted Dynamic Agricultural Landscapes. Journal of Ecology, 99, 1152-1161. https://doi.org/10.1111/j.1365-2745.2011.01840.x

[16] Valdés, A., Lenoir, J., Gallet-Moron, E., Andrieu, E., Brunet, J., Chabrerie, O., De Smedt, P., et al. (2015) The Contribution of Patch-Scale Conditions Is Greater than That of Macroclimate in Explaining Local Plant Diversity in Fragmented Forests across Europe. Global Ecology and Biogeography, 24, 1094-1105. https://doi.org/10.1111/geb.12345

[17] De Frenne, P., Graae, B.J., Rodríguez-Sánchez, F., Kolb, A., Chabrerie, O., Decocq, G., Gruwez, R., et al. (2013) Latitudinal Gradients as Natural Laboratories to Infer Species' Responses to Temperature. Journal of Ecology, 101, 784-795. https://doi.org/10.1111/1365-2745.12074

[18] Farina, A. (2019) Hybrid Nature: Effects on Environmental Fundamentals and Species' Semiosis. Biosemiotics, 1-20. https://doi.org/10.1007/s12304-019-09373-9

[19] Mercier, A., Ajzenberg, D., Devillard, S., Demar, M.P., De Thoisy, B., Bonnabau, H., 
Carme, B., et al. (2011) Human Impact on Genetic Diversity of Toxoplasma gondii: Example of the Anthropized Environment from French Guiana. Infection, Genetics and Evolution, 11, 1378-1387. https://doi.org/10.1016/j.meegid.2011.05.003

[20] Kalesnik, F. and Aceñolaza, P. (2008) Regional Distribution of Native and Exotic Species in Levees of the Lower Delta of the Paraná River. Acta Scientiarum. Biological Sciences, 30, 391-402. https://doi.org/10.4025/actascibiolsci.v30i4.5869

[21] Acevedo-Rodríguez, P. and Strong, M.T. (2008) Floristic Richness and Affinities in the West Indies. The Botanical Review, 74, 5-36.

https://doi.org/10.1007/s12229-008-9000-1

[22] Losos, J.B. (2010) Adaptive Radiation, Ecological Opportunity, and Evolutionary Determinism: American Society of Naturalists EO Wilson Award Address. The American Naturalist, 175, 623-639. https://doi.org/10.1086/652433

[23] Carmona, E.C., Ramírez, A.V. and Cano-Ortiz, A. (2010) Contribution to the Biogeography of the Hispaniola (Dominican Republic, Haiti). Acta Botanica Gallica, 157, 581-598. https://doi.org/10.1080/12538078.2010.10516233

[24] Maunder, M., Abdo, M., Berazain, R., Clubbe, C., Jiménez, F., Leiva, A., Francisco-Ortega, J., et al. (2011) The Plants of the Caribbean Islands: A Review of the Biogeography, Diversity and Conservation of a Storm-Battered Biodiversity Hotspot. In: The Biology of Island Floras, Cambridge University Press, London, 154-178. https://doi.org/10.1017/CBO9780511844270.007

[25] Banda-Rodríguez, K., Weintritt, J. and Pennington, R.T. (2016) Caribbean Dry Forest Networking: An Opportunity for Conservation. Caribbean Naturalist, No. 1, 63-72.

[26] DeWalt, S.J., Ickes, K. and James, A. (2016) Forest and Community Structure of Tropical Sub-Montane Rain Forests on the Island of Dominica, Lesser Antilles. Caribbean Naturalist, No. 1, 116-137.

[27] Rojas-Sandoval, J., Tremblay, R.L., Acevedo-Rodríguez, P. and Díaz-Soltero, H. (2017) Invasive Plant Species in the West Indies: Geographical, Ecological, and Floristic Insights. Ecology and Evolution, 7, 4522-4533.

https://doi.org/10.1002/ece3.2984

[28] Weaver, P.L. (2010) Tree Species Distribution and Forest Structure along Environmental Gradients in the Dwarf Forest of the Luquillo Mountains of Puerto Rico. Bois et Forets des Tropiques, 306, 33-44. https://doi.org/10.19182/bft2010.306.a20429

[29] Germa, A., Quidelleur, X., Labanieh, S., Lahitte, P. and Chauvel, C. (2010) The Eruptive History of Morne Jacob Volcano (Martinique Island, French West Indies): Geochronology, Geomorphology and Geochemistry of the Earliest Volcanism in the Recent Lesser Antilles Arc. Journal of Volcanology and Geothermal Research, 198, 297-310. https://doi.org/10.1016/j.jvolgeores.2010.09.013

[30] Allen, C.D. (2017) Landscapes and Landforms of the Lesser Antilles. Springer, New York. https://doi.org/10.1007/978-3-319-55787-8

[31] Mantran, M., Hamparian, R. and Bouchereau, J.L. (2009) Geomorphology and Hydrology of the Manche-à-Eau Lagoon (Guadeloupe, French Islands). Geomorphology: Relief, Process, Environment, 15, 199-210. https://doi.org/10.4000/geomorphologie.7606

[32] Cambers, G. (2005) Caribbean Islands, Coastal Ecology and Geomorphology. In: Schwartz, M.L., Ed., Encyclopedia of Coastal Science, Springer, Berlin, 221-226.

[33] Alexandre, H., Faure, J., Ginzbarg, S., Clark, J. and Joly, S. (2017) Bioclimatic Niches are Conserved and Unrelated to Pollination Syndromes in Antillean Gesneria- 
ceae. Royal Society Open Science, 4, Article ID: 170293.

https://doi.org/10.1098/rsos.170293

[34] Franklin, J., Andrade, R., Daniels, M.L., Fairbairn, P., Fandino, M.C., Gillespie, T.W., Kelly, D.L., et al. (2018) Geographical Ecology of Dry Forest Tree Communities in the West Indies. Journal of Biogeography, 45, 1168-1181.

https://doi.org/10.1111/jbi.13198

[35] Ewel, J.J. and Whitmore, J.L. (1973) The Ecological Life Zones of Puerto Rico and the US Virgin Islands. USDA Forest Service, Institute of Tropical Forestry, Research Paper ITF-018, 18.

[36] Beard, J.S. (1949) The Natural Vegetation of Windward and Leeward Islands. $O X^{-}$ ford Forestry Mem., No. 21, 192 p.

[37] Fiard, J.P. (1994) The Forests of Northern Pelée Mountain and the Volcanic Structures of Mont-Conil Peak and Le Morne-Sibérie. University Diploma in Tropical Phyto-Ecology and Island Development. University of the Antilles and Guyana, 595 p.

[38] Howard, R.A. (1950) The Vegetation of the Grenadines, Windward Islands, British West Indies. Contributions from the Gray Herbarium of Havard University, $n$ CLXXIV, Gray Herbarium, Cambrige.

[39] Joseph, P. (1997) Dynamics, Plant Ecophysiology in Dry Bioclimate in Martinique. Doctoral Thesis New Regime, University of the Antilles and Guyana, 941 p., Appendices, $111 \mathrm{p}$.

[40] Stehle, H. (1947) The Forest Vegetation of the Caribbean Archipelago. Faculty of Sciences, Montpellier, $548 \mathrm{p}$.

[41] Tallis, H., Levin, P.S., Ruckelshaus, M., Lester, S.E., McLeod, K.L., Fluharty, D.L. and Halpern, B.S. (2010) The Many Faces of Ecosystem-Based Management: Making the Process Work Today in Real Places. Marine Policy, 34, 340-348. https://doi.org/10.1016/j.marpol.2009.08.003

[42] Doré, J.C. and Ojasoo, T. (2001) How to Analyze Publication Time Trends by Correspondence Factor Analysis: Analysis of Publications by 48 Countries in 18 Disciplines over 12 Years. Journal of the American Society for Information Science and Technology, 52, 763-769. https://doi.org/10.1002/asi.1130

[43] Thorson, J.T., Scheuerell, M.D., Shelton, A.O., See, K.E., Skaug, H.J. and Kristensen, K. (2015) Spatial Factor Analysis: A New Tool for Estimating Joint Species Distributions and Correlations in Species Range. Methods in Ecology and Evolution, 6, 627-637. https://doi.org/10.1111/2041-210X.12359

[44] Kolahi, M. and Atri, M. (2014) The Effect of Ecological Factors on Vegetation in Hamedan Alvand Region (Iran). International Journal of Farming and Allied Sciences, 3, 489-496.

[45] Saima, S., Altaf, A., Faiz, M.H., Shahnaz, F. and Wu, G. (2018) Vegetation Patterns and Composition of Mixed Coniferous Forests along an Altitudinal Gradient in the Western Himalayas of Pakistan. Austrian Journal of Forest Science, 135, 159-180.

[46] Kawai, T. and Tokeshi, M. (2007) Testing the Facilitation-Competition Paradigm under the Stress-Gradient Hypothesis: Decoupling Multiple Stress Factors. Proceedings of the Royal Society B: Biological Sciences, 274, 2503-2508. https://doi.org/10.1098/rspb.2007.0871

[47] Berner, D. and Thibert-Plante, X. (2015) How Mechanisms of Habitat Preference Evolve and Promote Divergence with Gene Flow. Journal of Evolutionary Biology, 28, 1641-1655. https://doi.org/10.1111/jeb.12683

[48] Miller, T.E., Gornish, E.S. and Buckley, H.L. (2010) Climate and Coastal Dune Ve- 
getation: Disturbance, Recovery, and Succession. Plant Ecology, 206, 97. https://doi.org/10.1007/s11258-009-9626-Z

[49] Orrock, J.L. and Witter, M.S. (2010) Multiple Drivers of Apparent Competition Reduce Re-Establishment of a Native Plant in Invaded Habitats. Oikos, 119, 101-108. https://doi.org/10.1111/j.1600-0706.2009.17831.x 
Annex 1. List of Species Abbreviations

\begin{tabular}{|c|c|c|c|c|c|}
\hline Acacia macracantha & Ama & Chiococca alba & Chio & Exostema caribaeum & Eeu \\
\hline Acacia nilotica & $A n$ & Chionanthus compacta & $C c$ & $\begin{array}{c}\text { Exostema } \\
\text { sanctae-luciae }\end{array}$ & Esl \\
\hline Acacia $s p$ & $A s p$ & Chrysobalanus icaco & Chry & Exothea paniculata & Eth \\
\hline Acacia tamarindifolia & At & $\begin{array}{l}\text { Chrysophyllum } \\
\text { argenteum }\end{array}$ & Cge & Faramea occidentalis & Fo \\
\hline Acrocomia aculeata & $\mathrm{Aa}$ & $\begin{array}{l}\text { Cinnamomum } \\
\text { elongatum }\end{array}$ & Cin & Ficus citrifolia & Fc \\
\hline $\begin{array}{l}\text { Actinostemon } \\
\text { caribaeus }\end{array}$ & Ac & Citharexylum spinosum & Cit & Ficus nymphaeifolia & $F n$ \\
\hline $\begin{array}{c}\text { Aegiphila } \\
\text { martinicensis }\end{array}$ & Aem & $\begin{array}{l}\text { Clerodendron } \\
\text { aculeatum }\end{array}$ & Cle & Ficus trigonata & Ft \\
\hline Agave sp. & Ags & Clusia major & Cjo & Forestiera rhamnifolia & $F r$ \\
\hline Aiphanes erosa & $A e$ & Coccoloba coronata & Cta & Garcinia humilis & Gh \\
\hline Aiphanes minima & $A m i$ & Coccoloba diversifolia & $C d i$ & Genipa americana & Ga \\
\hline Albizia caribaea & Alc & Coccoloba krugii & $C k r$ & Guarea macrophylla & $G m$ \\
\hline Albizzia lebbeck & All & Coccoloba pubescens & Cpu & Guazuma ulmifolia & Gu \\
\hline Allophyllus racemosus & $A r$ & Coccoloba swartzii & $C s w$ & Guettarda odorata & Go \\
\hline Amphitecna latifolia & $A m l$ & Coccoloba uvifera & $\mathrm{Cuv}$ & Guettarda scabra & Gs \\
\hline Amyris elemifera & Amy & Coccoloba venosa & Cve & Gyminda latifolia & $G 1$ \\
\hline $\begin{array}{l}\text { Anacardium } \\
\text { occidentale }\end{array}$ & $A o$ & $\begin{array}{c}\text { Coccothrinax } \\
\text { barbadensis }\end{array}$ & Cis & $\begin{array}{l}\text { Haemathoxylon } \\
\text { campechianum }\end{array}$ & $H c$ \\
\hline Andira inermis & And & Colubrina arborescens & Cns & Hernendia sonora & $H s$ \\
\hline Annona muricata & Anm & Comocladia dodeanea & Cdo & $\begin{array}{c}\text { Hippomane } \\
\text { mancinella }\end{array}$ & $H m$ \\
\hline Annona squamosa & Asq & Conostegia calyptrata & Cgia & $\begin{array}{l}\text { Homalium } \\
\text { racemosum }\end{array}$ & $H r$ \\
\hline Antirhea coriacea & Ant & Cordia alliodora & Call & Hura crepitans & $H c$ \\
\hline Ardisia obovata & Ard & Cordia collococca & Ccol & Hymenea courbaril & $H i$ \\
\hline Bontia daphnoides & $B d$ & Cordia curassavica & $C c u$ & Ilex nitida & In \\
\hline Bourreria succulenta & Bs & Cordia glabra & Gga & Inga laurina & Il \\
\hline Bromelia plumieri & $B p$ & Cordia globosa & Cglo & Ixora ferrea & If \\
\hline Brosimum alicastrum & $B a$ & Cordia martinicensis & $\mathrm{Cm}$ & Jacquinia armilaris & Ja \\
\hline Brunfelsia americana & $B r$ & Cordia obliqua & $C o b$ & $\begin{array}{l}\text { Krugiodendron } \\
\quad \text { ferreum }\end{array}$ & $K f$ \\
\hline $\begin{array}{l}\text { Buchenavia tetraphyl- } \\
\text { la }\end{array}$ & $B u$ & Cordia sulcata & Csu & Laetia thamnia & $L t$ \\
\hline Bucida buceras & $B b$ & Cornutia pyramidata & $C p y$ & Lantana camara & $L c$ \\
\hline Bunchosia glandulosa & $B g$ & Crateva tapia & $C t p$ & Lantana involucrata & $L i$ \\
\hline Bursera simaruba & $B s i$ & Crossopetalum rhacoma & Crh & $\begin{array}{c}\text { Leucaena } \\
\text { leucocephala }\end{array}$ & $L I$ \\
\hline Byrsonima lucida & By & Croton astroides & $\mathrm{Coi}$ & Licania ternatensis & Ls \\
\hline Byrsonima spicata & Bys & Croton corylifolius & Cfo & Licaria sericea & Le \\
\hline Caesalpinia coriaria & $\mathrm{Cco}$ & Croton flavens & Cfs & $\begin{array}{c}\text { Lonchocarpus } \\
\text { pentaphyllus }\left(^{*}\right)\end{array}$ & $L p$ \\
\hline
\end{tabular}




\section{Continued}

\begin{tabular}{|c|c|c|c|c|c|}
\hline Calliandra prupurea & $C p$ & Croton sp. & $C s p$ & $\begin{array}{c}\text { Lonchocarpus } \\
\text { violaceus }\end{array}$ & $L_{V}$ \\
\hline Calliandra slaneae & Csl & Cupania americana & Cna & Maclura tinctoria & Mt \\
\hline Calliandra tergemina & $C t$ & Daphnopsis americana & $\mathrm{Da}$ & Malpighia emarginata & $\mathrm{Me}$ \\
\hline Calophyllum calaba & $\mathrm{Ca}$ & Diospyros inconstans & $D i$ & Malpighia glabra & $M g$ \\
\hline Calotropis procera & $C p r$ & Dodonaea viscosa & $D_{V}$ & Malpighia linearis & $M I$ \\
\hline Canella winterana & $C w$ & Erithalis fruticosa & $E f$ & Mangifera indica & Mi \\
\hline Capparis baducca & $C b$ & Erithalis odorifera & Eo & Manilkara bidentata & $M b$ \\
\hline $\begin{array}{c}\text { Capparis } \\
\text { cynophallophoa }\end{array}$ & Ccy & Erythroxylon havanense & $E h$ & Margaritaria nobilis & $M n$ \\
\hline Capparis flexuosa & $C f$ & Eugenia (Bahamensis) & $E b$ & Maytenus laevigata & $M a$ \\
\hline Capparis hastata & $C h$ & Eugenia axillaris & $E a$ & Melocactus intortus & $M t i$ \\
\hline Capparis indica & $C i$ & $\begin{array}{c}\text { Eugenia } \\
\text { chrysobalanoides }\end{array}$ & Ey & Melochia tomentosa & $M h$ \\
\hline Capparis odoratissima & Co & Eugenia confusa & Efu & Melococcus bijugatus & $M j$ \\
\hline Casearia decandra & $C d$ & Eugenia cordata & $E c$ & Morinda citrifolia & $M f$ \\
\hline Casearia guianensis & $\mathrm{Cg}$ & Eugenia gregii & $E g$ & Morisonia americana & $M m$ \\
\hline $\begin{array}{l}\text { Cassipourea } \\
\text { guianensis }\end{array}$ & Cas & Eugenia hodgei & $E u$ & Myrcia citrifolia & $M c i$ \\
\hline Catesbea melanocarpa & Cmel & Eugenia lambertiana & Ela & Myrcia fallax & $M f a$ \\
\hline Cecropia schreberiana & Csc & Eugenia ligustrina & Eli & Myrciaria floribunda & $M f l$ \\
\hline Cedrela odorata & Cod & Eugenia monticola & Emo & Ocotea cernua & $O c$ \\
\hline Ceiba pentandra & $\mathrm{Cei}$ & Eugenia oerstedeana & Eoe & Ocotea coriacea & $\mathrm{Oa}$ \\
\hline Celtis iguanea & $\mathrm{Cig}$ & Eugenia pseudopsidium & Eps & Ocotea eggersiana & Oe \\
\hline Cestrum sp. & $C s p$ & Eugenia rhombea & Erh & Ocotea glabra & $O g$ \\
\hline $\begin{array}{c}\text { Chamaecrista } \\
\text { glandulosa var. } \\
\text { swartzii }\end{array}$ & $C g I$ & Eugenia tapacumensis & Etp & Ocotea leucoxylon & $\mathrm{OI}$ \\
\hline Ocotea patens & $O p$ & Tecoma stans & $T s$ & & \\
\hline Opuntia dillenii & Od & $\begin{array}{l}\text { Ternstroenia } \\
\text { peduncularis }\end{array}$ & $T p$ & & \\
\hline Ormosia monosperma & Om & Tetrazygia angustifolia & $\mathrm{Ta}$ & & \\
\hline Ouratea guildingii & Ogg & Trichilia hirta & $T t$ & & \\
\hline Persea americana & $\mathrm{Pa}$ & Triphasia trifolia & $T f$ & & \\
\hline $\begin{array}{c}\text { Phyllanthus } \\
\text { (angustifolius) }\end{array}$ & Ps & $\begin{array}{c}\text { Vangueria } \\
\text { madagascariensis }\end{array}$ & $V m$ & & \\
\hline Picramnia pentandra & $P p$ & Vernonia albicaulis & $V a$ & & \\
\hline Pilocarpus racemosus & $\operatorname{Pr}$ & Vernonia arborescens & $V s$ & & \\
\hline Pilosocereus royeni & $P i$ & $\begin{array}{c}\text { Zanthoxylum } \\
\text { caribaeum }\end{array}$ & $Z c$ & & \\
\hline Pimenta racemosa & $P m$ & Zanthoxylum flavum & $Z f$ & & \\
\hline $\begin{array}{c}\text { Piscidia } \\
\text { carthagenensis }\end{array}$ & $P h$ & $\begin{array}{l}\text { Zanthoxylum } \\
\text { martinicense }\end{array}$ & $Z m$ & & \\
\hline
\end{tabular}




\section{Continued}

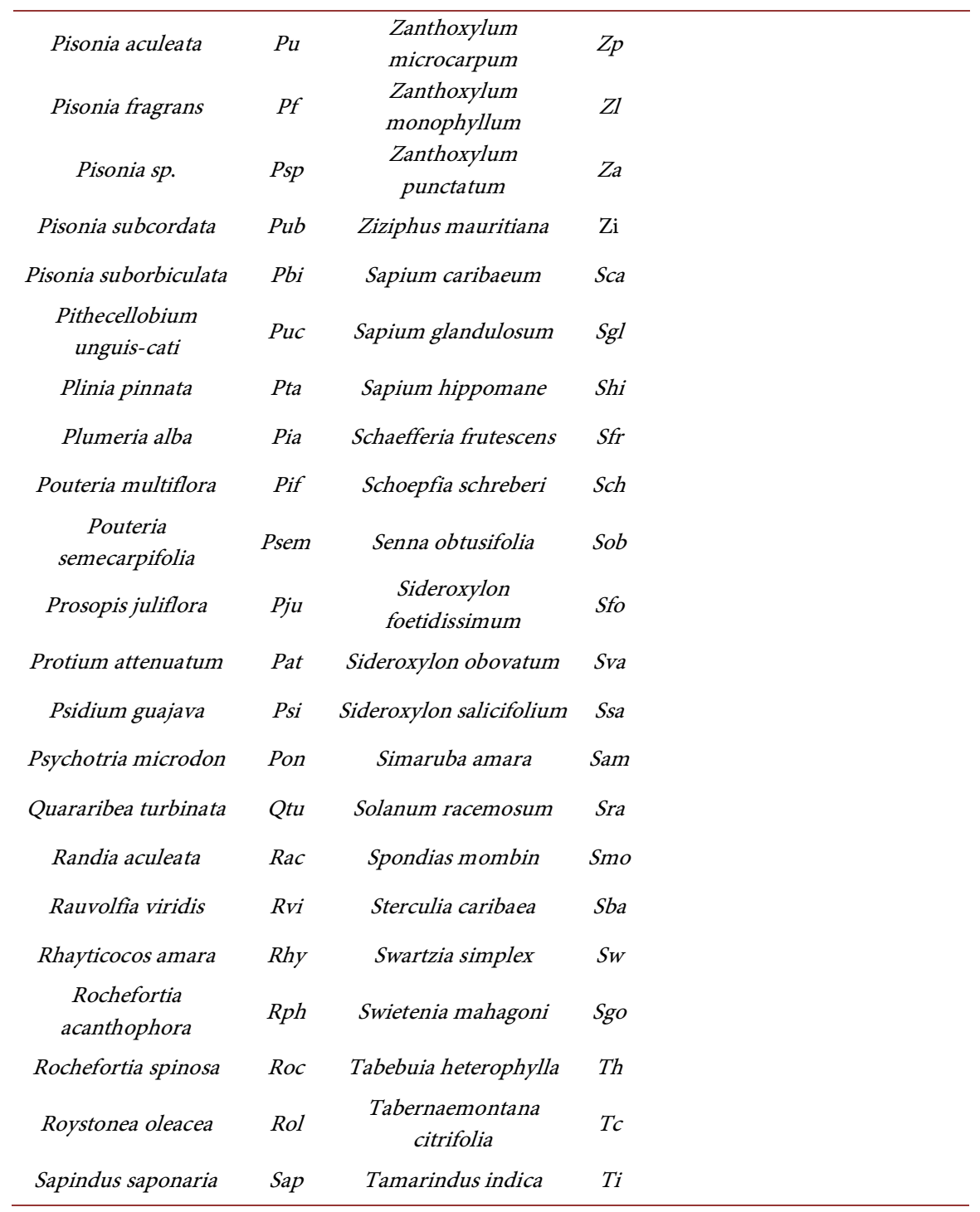

